\title{
Charge completeness and the massless charge lattice in F-theory models of supergravity
}

\author{
David R. Morrison ${ }^{a}$ and Washington Taylor ${ }^{b}$ \\ ${ }^{a}$ Departments of Mathematics and Physics, University of California, Santa Barbara, \\ 1210 Cheadle Hall, Santa Barbara, CA 93106, U.S.A. \\ ${ }^{b}$ Center for Theoretical Physics, Department of Physics, Massachusetts Institute of Technology, \\ 77 Massachusetts Avenue, Cambridge, MA 02139, U.S.A. \\ E-mail: drm@math.ucsb.edu, wati@mit.edu
}

ABSTRACT: We prove that, for every 6D supergravity theory that has an F-theory description, the property of charge completeness for the connected component of the gauge group (meaning that all charges in the corresponding charge lattice are realized by massive or massless states in the theory) is equivalent to a standard assumption made in F-theory for how geometry encodes the global gauge theory by means of the Mordell-Weil group of the elliptic fibration. This result also holds in 4D F-theory constructions for the parts of the gauge group that come from sections and from 7-branes. We find that in many $6 \mathrm{D} \mathrm{F}$ theory models the full charge lattice of the theory is generated by massless charged states; this occurs for each gauge factor where the associated anomaly coefficient satisfies a simple positivity condition. We describe many of the cases where this massless charge sufficiency condition holds, as well as exceptions where the positivity condition fails, and analyze the related global structure of the gauge group and associated Mordell-Weil torsion in explicit F-theory models.

KEYWORDS: F-Theory, Superstring Vacua

ArXiv EPrint: 2108.02309 


\section{Contents}

1 Introduction $\quad 2$

2 Quick review of 6D supergravity and F-theory realizations 4

2.1 Anomaly conditions 4

$2.2 \quad$ F-theory models of $6 \mathrm{D}$ and $4 \mathrm{D}$ supergravity theories 6

3 The completeness hypothesis and massless charge sufficiency 8

3.1 Statement of conditions 8

3.2 Proof of charge completeness hypothesis in F-theory 8

$\begin{array}{ll}3.3 \text { Massless charge sufficiency in 6D } & 10\end{array}$

4 Examples: massless charge sufficiency and Mordell-Weil torsion for non$\begin{array}{ll}\text { abelian gauge groups } & \mathbf{1 2}\end{array}$

$\begin{array}{lll}4.1 & \mathbb{Z}_{2} \text { torsion } & 13\end{array}$

4.1.1 General Weierstrass models with $\mathbb{Z}_{2}$ torsion 13

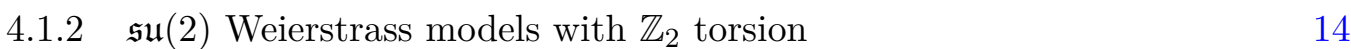

$\begin{array}{lll}\text { 4.1.3 } & \mathbb{Z}_{2} \text { torsion and larger } \mathrm{SU}(N) \text { and } \operatorname{Sp}(N) \text { groups } & 16\end{array}$

$\begin{array}{lll}\text { 4.1.4 } & E_{7} \text { gauge group } & 18\end{array}$

$\begin{array}{lll}\text { 4.1.5 } & \mathrm{SO}(N) \text { and } \mathbb{Z}_{2} \text { torsion } & 19\end{array}$

$4.2 \mathbb{Z}_{3}$ torsion: $\mathrm{SU}(3), E_{6}, \mathrm{SU}(6)$ (but not $\mathrm{SU}(9)$ ) 21

4.3 Higher torsion groups 23

$\begin{array}{lll}4.3 .1 & \mathbb{Z}_{4} & 23\end{array}$

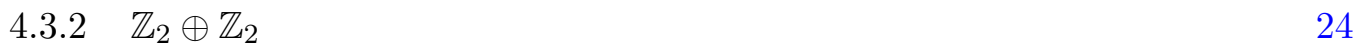

$\begin{array}{lll}\text { 4.3.3 } & \text { Other discrete torsion groups } & 25\end{array}$

$5 \quad \mathrm{U}(1)$ gauge factors $\quad \mathbf{2 6}$

$5.1 \mathrm{U}(1)$ and "charge 2" matter 26

$\begin{array}{lll}5.2 & \text { Other aspects of } \mathrm{U}(1) \text { factors } & 29\end{array}$

$6 \quad$ Exceptions to massless charge sufficiency $\quad 29$

6.1 Non-Higgsable nonabelian factors: exceptional cases with $-a \cdot b<0 \quad 29$

6.2 Geometry of exceptional cases with $-a \cdot b=b \cdot b=0$

6.3 Non-Higgsable U(1) factors 32

6.4 Nonabelian factors with a single adjoint matter field 33

$\begin{array}{lll}7 & \text { Conclusions } & 34\end{array}$

A The fundamental group of the gauge group 35 


\section{Introduction}

String theory, in its many avatars, gives rise to a wide range of vacuum solutions in various dimensions with a great variety of different gauge groups and matter content coupled to quantum gravity. This large range of solutions has led to the suggestion that essentially "anything goes", and that virtually any consistent quantum field theory can be found coupled to quantum gravity in some string theory. In fact, however, quantum gravity and string theory place strong constraints on the structure of theories with a consistent UV completion.

This tension between the constraints coming from string theory and consistency conditions apparent for a low-energy quantum field theory coupled to gravity has been present since the early days of string theory. A simple example is the set of consistent $\mathcal{N}=1$ supersymmetric gravity theories in ten dimensions. The seminal work of Green and Schwarz [1] showed that all such supergravity theories were inconsistent except those with gauge groups $\operatorname{Spin}(32) / \mathbb{Z}_{2}, E_{8} \times E_{8}, E_{8} \times \mathrm{U}(1)^{248}$ and $\mathrm{U}(1)^{496}$. At the time only the first of these gauge groups was known to arise in string theory. Motivated by this result, the "string quartet" identified the heterotic $E_{8} \times E_{8}$ string theory [2,3]. More recently, Vafa coined the term "swampland" [4] to describe those theories that appear to be consistent under all known quantum consistency conditions but that are not (known to be) realized in string theory, and pointed out that, at the time this term was coined, the 10D theories with gauge groups $E_{8} \times \mathrm{U}(1)^{248}$ and $\mathrm{U}(1)^{496}$ appeared to be in the swampland. In [5], it was shown that in fact these theories cannot be simultaneously compatible with supersymmetry and gauge invariance. The same conclusion was derived from a string worldsheet point of view in [6]. Thus, for minimally supersymmetric 10D theories of gravity coupled to gauge groups, at least at the level of massless spectra the swampland is empty. In fewer than ten space-time dimensions, however, the set of theories with known realizations in string theory is a proper subset of those that satisfy all known consistency conditions, and the ongoing study of the difference between these two sets has been a productive focus of much recent research effort; an overview of recent conjectures and developments related to the swampland is given in [7].

Six-dimensional $\mathcal{N}=(1,0)$ supergravity theories present a particularly interesting domain in which to investigate "swampland" related questions. As in ten dimensions, the requirement of gravitational and mixed gauge-gravitational anomaly cancellation provides strong constraints on the set of possibly consistent $6 \mathrm{D}$ theories. At the same time, string constructions of $6 \mathrm{D}$ supergravity theories are fairly well understood, particularly in the context of F-theory [8-10]. The set of $6 \mathrm{D}$ supergravity theories that can be realized through known string constructions essentially forms one large moduli space, with different branches connected by various kinds of geometric transitions. ${ }^{1}$ It was conjectured in [12] that, as in $10 \mathrm{D}$, it may be possible to show that every quantum-consistent massless $6 \mathrm{D}$ supergravity spectrum is realized in string theory. The close connection between the structure of $6 \mathrm{D}$ supergravity and F-theory (see e.g. [13]) gives many insights into these theories, but at this

\footnotetext{
${ }^{1}$ Note, it has not been shown how the theories in [11] are connected to the connected moduli space of standard F-theory constructions.
} 
time there are still substantial classes of apparently consistent $6 \mathrm{D}$ supergravity theories without an F-theory realization; for example, there is an infinite class of theories with no tensor multiplets, a single $\mathrm{U}(1)$ gauge factor, and matter fields with arbitrarily large $\mathrm{U}(1)$ charges that cannot be realized in F-theory but that satisfy anomaly cancellation and all other known quantum consistency conditions on low-energy 6D field theories coupled to gravity [14]. Other aspects of the 6D supergravity swampland have been explored in a variety of papers, including $[6,11,15-20]$.

Another aspect of "swampland" related questions involves certain features that appear to be typical of all string constructions (in a variety of dimensions), and for some of which there are heuristic arguments involving quantum gravity features such as black hole information and radiation. Some analysis and conjectures based on features of this type were given in [21-23]. One particular feature of string theory is the general aspect that any time there is a symmetry, with an associated gauge or higher form field, charged objects appear in the theory, and these objects saturate the allowed space of charges in most (perhaps all) known string solutions. This principle was part of the philosophy underlying the development of D-branes [24], which are the charged objects associated with the Ramond-Ramond fields of type II string theory. This notion of "charge completeness" and the related idea that theories of quantum gravity cannot have global symmetries, only gauge symmetries, were articulated clearly in [25]. For theories in anti-de Sitter space with holographic dual descriptions, these conjectures were recently proven by Harlow and Ooguri [26].

In this paper we address the charge completeness question in the context of $6 \mathrm{D}$ and $4 \mathrm{D}$ supergravity theories. We prove using Poincaré duality that for $6 \mathrm{D} \mathcal{N}=(1,0)$ supergravity theories that arise from F-theory, the charge completeness hypothesis for the connected component of the gauge group is equivalent to the standard assumption made in F-theory for how the global structure of the gauge group is encoded in geometry. ${ }^{2}$ We show that the same result also holds in 4D F-theory constructions for the part of the gauge group that is realized through 7-branes and sections. (Note that in $6 \mathrm{D}$ all of these supergravity theories live in flat space-time, so that the proof of Harlow and Ooguri is not applicable.)

We furthermore observe that for many $6 \mathrm{D} \mathcal{N}=(1,0)$ supergravity theories a stronger condition holds: in these models the charge lattice is completely determined by the set of massless fields in the theory. We identify a simple positivity condition on the anomaly coefficients of the gauge factors that seems to be sufficient to imply this "massless charge sufficiency" condition. We give a large set of examples of 6D supergravity theories with connected nonabelian and abelian gauge groups where this condition holds, and analyze some of the cases where it does not. Mordell-Weil torsion and the global structure of the gauge group play an important role in understanding these structures.

The structure of the paper is as follows. We begin in section 2 with a very brief review of $6 \mathrm{D} \mathcal{N}=(1,0)$ supergravity theories and their realization in F-theory. In section 3 we

\footnotetext{
${ }^{2}$ As described in more detail in section 2.2 and the appendix, the "standard assumption" [27-29] relates the fundamental group $\pi_{1}(G)$ of the gauge group $G$ to the group of sections (the "Mordell-Weil group") of the elliptic fibration used in the F-theory construction, by considering which representations of the corresponding Lie algebra can occur in the presence of Mordell-Weil group elements.
} 
state the completeness and massless charge sufficiency conditions. We prove a version of the completeness hypothesis for those theories with an F-theory realization, and conjecture that the massless charge sufficiency condition holds in all but certain special exceptional circumstances. In section 4 , we give a variety of examples of $6 \mathrm{D} \mathcal{N}=(1,0)$ supergravity theories with nonabelian gauge groups where the gauge algebra does not uniquely determine the global structure of the gauge group. In many of these theories we prove that the massless charges in the theory are sufficient to generate the full charge lattice, and explicitly describe the connection in these theories between Mordell-Weil torsion in the F-theory model and the fundamental group of the gauge group of the associated $6 \mathrm{D}$ supergravity theory. In section 5 we consider an example with an abelian gauge group, and in section 6 we describe several of the exceptional classes of models where the massless charge spectrum is not sufficient to determine the full charge lattice of the theory. Section 7 contains some concluding remarks. We include an appendix with a discussion of the topology of the gauge group and its relation to representation theory.

Some recent papers touch on issues closely related to the subject and techniques of this work. The structure of discrete 1-form symmetries in $6 \mathrm{D}$ supergravity theories with Mordell-Weil torsion like many of the models studied here was investigated in [30]. In [31] related aspects of the global structure of the gauge group for 8D F-theory models have been investigated. In [19], a general characterization is given of a large set of $6 \mathrm{D}$ supergravity models that appear to be compatible with known quantum consistency conditions but lack an F-theory realization. Such models violate an automatic enhancement condition that seems to hold for all theories that come from F-theory. The automatic enhancement conjecture made in that paper is closely related in some cases to the massless charge sufficiency conjecture presented here, as discussed further in that paper; the analysis of that paper was inspired in part by some examples encountered in this work.

\section{Quick review of $6 \mathrm{D}$ supergravity and F-theory realizations}

We begin by briefly reviewing the structure of $6 \mathrm{D} \mathcal{N}=(1,0)$ supergravity theories and their realizations in F-theory

\subsection{Anomaly conditions}

In general a $6 \mathrm{D} \mathcal{N}=(1,0)$ supergravity theory has $T$ tensor multiplets, $V=\operatorname{dim} G=$ $\operatorname{dim} G^{0}$ vector multiplets where the connected component ${ }^{3} G^{0}$ of the gauge group $G$ can generally have both nonabelian and abelian factors, taking the form ${ }^{4} G^{0}=\left(G_{0} \times \mathrm{U}(1)^{r}\right) / \Xi$ (with $G_{0}$ simply connected and $\Xi$ a finite subgroup of the center of $\left.G_{0} \times \mathrm{U}(1)^{r}\right)$, and $H$ matter hypermultiplets, which can be neutral or charged under the gauge group. The $6 \mathrm{D}$ gravitational, nonabelian gauge, and gravitational-nonabelian gauge anomaly cancellation

\footnotetext{
${ }^{3}$ The gauge group $G$ can also have a disconnected part $G / G^{0}$ but that will not concern us in this paper. In fact, $\pi_{1}(G)=\pi_{1}\left(G^{0}\right)$ so the condition as we have stated it is unchanged by passing from $G$ to $G^{0}$.

${ }^{4}$ See the appendix for more details about how this form is arrived at.
} 
conditions [32, 33] can be written in the form [13]

$$
\begin{aligned}
H-V+29 T & =273 \\
0 & =B_{\mathrm{adj}}^{i}-\sum_{R} x_{R}^{i} B_{R}^{i} \\
a \cdot a & =9-T \\
-a \cdot b_{i} & =\frac{1}{6} \lambda_{i}\left(\sum_{R} x_{R}^{i} A_{R}^{i}-A_{\mathrm{adj}}^{i}\right) \\
b_{i} \cdot b_{i} & =\frac{1}{3} \lambda_{i}^{2}\left(\sum_{R} x_{R}^{i} C_{R}^{i}-C_{\mathrm{adj}}^{i}\right) \\
b_{i} \cdot b_{j} & =\lambda_{i} \lambda_{j} \sum_{R S} x_{R S}^{i j} A_{R}^{i} A_{S}^{j} .
\end{aligned}
$$

Here, $a, b_{i}$ are the coefficients associated with the Green-Schwarz terms $a \cdot B \wedge R \wedge R, b_{i}$. $B \wedge F_{i} \wedge F_{i}$ in the action, with $F_{i}$ the $i$ th nonabelian field strength; these coefficients lie in a lattice $\Gamma$ of signature $(1, T)$. The quantities $x_{R}^{i}$ denote the number of hypermultiplets transforming in representation $R$ under gauge group factor $G_{i}$, while the quantities $x_{R S}^{i j}$ denote the number of hypermultiplets transforming in representations $R, S$ under the factors $G_{i}, G_{j}$. The coefficients $A_{R}, B_{R}, C_{R}$ are defined through

$$
\begin{aligned}
& \operatorname{tr}_{R} F^{2}=A_{R} \operatorname{tr} F^{2} \\
& \operatorname{tr}_{R} F^{4}=B_{R} \operatorname{tr} F^{4}+C_{R}\left(\operatorname{tr} F^{2}\right)^{2},
\end{aligned}
$$

where $F$ is a field strength for the appropriate group factor and $t r$ is a normalized trace. Tables of the coefficients $A_{R}, B_{R}, C_{R}$ can be found in, e.g., [34-36] (see also [37, appendix C]). Note that for groups with no quartic Casimir (like $\mathrm{SU}(2)$, $\mathrm{SU}(3)$, and the exceptional groups), $B_{R}=0$ and the condition (2.2) is trivial. As a simple example of the anomaly conditions, for $\mathrm{SU}(2)$ we have $\lambda=1$, and the anomaly coefficients $A_{R}, C_{R}$ for the fundamental and adjoint representations are $1,1 / 2$ and 4,8 respectively.

For a theory with a single $\mathrm{U}(1)$ gauge factor, the associated gauge-gravitational and pure gauge anomaly conditions are

$$
\begin{aligned}
-a \cdot \tilde{b} & =\frac{1}{6} \sum_{q>0} x_{q} q^{2}, \\
\tilde{b} \cdot \tilde{b} & =\frac{1}{3} \sum_{q>0} x_{q} q^{4} .
\end{aligned}
$$

Here, $\tilde{b}$ is the $\mathrm{U}(1)$ Green-Schwarz anomaly coefficient and $x_{q}$ is the number of matter hypermultiplets of charge $q$ under the $\mathrm{U}(1)$. More details on anomaly conditions for theories with multiple $\mathrm{U}(1)$ and mixed abelian-nonabelian gauge groups, can be found in [34, 38, 39]. Some constraints on the anomaly coefficients $a, b, \tilde{b}$ are determined in $[13,16,40]$. 


\section{$2.2 \quad$ F-theory models of $6 \mathrm{D}$ and $4 \mathrm{D}$ supergravity theories}

A large class of $6 \mathrm{D}$ and $4 \mathrm{D}$ supergravity theories can be realized by an F-theory compactification $^{5}$ using an elliptic Calabi-Yau threefold or fourfold $X$. Such an F-theory compactification is defined ${ }^{6}$ through a Weierstrass model for the elliptic fibration on $X$,

$$
y^{2}=x^{3}+f x+g,
$$

where $f, g$ are sections of line bundles $\mathcal{O}\left(-4 K_{B}\right), \mathcal{O}\left(-6 K_{B}\right)$ over a base $B$ that is a complex surface for a $6 \mathrm{D}$ theory or a complex threefold for a $4 \mathrm{D}$ theory. Such an F-theory compactification can be thought of as a compactification of type IIB string theory on the base $B$ with an axiodilaton field $\tau$ that varies over $B$ and matches the ratio of periods $\tau$ of the elliptic curve defined through (2.10) at each point in $B$. More detailed introductions to F-theory models of $6 \mathrm{D}$ and $4 \mathrm{D}$ supergravity can be found in [44-48]; here we review a few of the main points. We focus principally on reviewing the structure of $6 \mathrm{D}$ F-theory models; 4D F-theory models are similar but have additional complications, as we discuss in the last paragraph of this section.

The nonabelian gauge algebra of a $6 \mathrm{D}$ supergravity theory realized through $\mathrm{F}$-theory is associated with Kodaira singularities $[49,50]$ in the elliptic fibration over complex curves $C_{i}$ in $B$. In the type IIB picture, these are $7+1$-dimensional objects (stacks of D7 branes or F-theory generalizations thereof) supporting nonabelian gauge factors. There is a close correspondence between the geometry of the elliptic Calabi-Yau threefold over $B$ and the physics of the resulting $6 \mathrm{D}$ theory. In particular, there is a direct mapping between the anomaly coefficients $a, b$ of the $6 \mathrm{D}$ theory and certain divisors in $B$. The string charge lattice $\Gamma$ of the $6 \mathrm{D}$ theory corresponds to the homology lattice $H_{1,1}(B, \mathbb{Z})$. The anomaly coefficient $a$ corresponds to the canonical class $K_{B}$, while $b_{i}$ corresponds to the class of the complex curve $C_{i}$. Abelian anomaly coefficients $\tilde{b}$ are described in a slightly more subtle but similar way in terms of specific divisors in $X$. The detailed relationships between the geometry of elliptic Calabi-Yau threefolds and the structure of the 6D theory, which relate geometric conditions to the $6 \mathrm{D}$ anomaly constraints, are described in further detail in [13, 16, 37, 39, 51-54]. F-theory in 6D is often analyzed as a limit of M-theory (see e.g. [55]), where in the M-theory picture the compactification manifold for the Coulomb branch is a smooth Calabi-Yau threefold $\tilde{X}$ associated with a resolution of the singularities of $X$.

While the Kodaira singularities in the elliptic fibration that arise at codimension one in the $\mathrm{F}$-theory base $B$ directly encode the gauge algebra of the corresponding $6 \mathrm{D}$ supergravity theory, the global structure of the gauge group is somewhat more subtle. Assume that a $6 \mathrm{D}$ supergravity theory has gauge group $G$ and comes from an F-theory Weierstrass model that defines an elliptic Calabi-Yau threefold $X$ with resolution $\tilde{X}$. The Cartan generators of the Lie algebra are associated with the (divisorial) components of the Kodaira fibers of

\footnotetext{
${ }^{5}$ For simplicity, we do not include the F-theory compactifications with "frozen" singularities studied in [11], nor do we allow background fluxes.

${ }^{6}$ In order to admit an effective description as a gauge theory coupled to supergravity, the singularities in the Weierstrass model cannot be too bad: along any locus of complex codimension two in the base, the multiplicities of $(f, g)$ must be less than $(4,6)$, or else the F-theory model will include a superconformal sector $[10,41-43]$.
} 
the codimension one singularities in the (resolved) fibration; these are divisors in $H^{1,1}(\tilde{X})$. Other divisors are associated with rational sections of the elliptic fibration; because rational sections can be "added" (using the group law of the elliptic fibration) the rational sections form a group ${ }^{7}$ called the Mordell-Weil group, which is a finitely generated abelian group and therefore has the form $\mathbb{Z}^{r} \times(M W)$ tors where $r$ is the rank of the group of sections and $(M W)_{\text {tors }}$ is the Mordell-Weil torsion. The standard assumption in F-theory is that the global structure of the $6 \mathrm{D}$ gauge group $G$ is determined by the condition that the MordellWeil group coincides with fundamental group of the gauge group (see [27] and footnote 4 in [28]), although there is no proof of this assumption. One of the primary results of this paper is to show that this assumption is equivalent to the charge completeness hypothesis for $6 \mathrm{D}$ F-theory models. In general, the connected part $G^{0}$ of the $6 \mathrm{D}$ gauge group $G$ is a compact Lie group, which can be written as a quotient $G^{0}=\left(G_{0} \times \mathrm{U}(1)^{r}\right) / \Xi$, where $G_{0}$ is simply connected and represents the nonabelian part of $G$, and $\Xi$ is a finite subgroup of the center of $G_{0} \times \mathrm{U}(1)^{r}$. The standard assumption regarding the global structure of the gauge group is then that $(1) \pi_{1}(G)_{\text {tors }}$ coincides with $\Xi \cap G_{0}=(M W)_{\text {tors }}$, and (2) the rank of the free part of the Mordell-Weil group measures the number of U(1) factors in the gauge group. Note that when $r=0, G^{0}=G_{0} / \Xi$, and $\Xi=(M W)_{\text {tors }}$; we consider a number of cases of this type in section 4 . On the other hand, when there are abelian factors there can be a nontrivial finite quotient even when $(M W)_{\text {tors }}$ is trivial; for example, models having such structure with group $\mathrm{U}(N)=(\mathrm{SU}(N) \times \mathrm{U}(1)) / \mathbb{Z}_{N}$ or with the standard model gauge group $(\mathrm{SU}(3) \times \mathrm{SU}(2) \times \mathrm{U}(1)) / \mathbb{Z}_{6}$ have been studied in various places in the literature [56-65].

The matter content of the 6D supergravity theory arising from an F-theory model arises from a combination of local singularity enhancements associated with intersecting 7-branes in the IIB picture and nonlocal structure associated with adjoint representations of the gauge group [10, 66-69]. We restrict attention in this paper to standard matter representations that can be realized through local enhancements of the Kodaira singularity type, and consider only briefly cases with local singularities in the elliptic fibration at codimension two where $(f, g)$ vanish to orders at least $(4,6)$; such singularities have no direct Calabi-Yau resolution preserving a flat elliptic fibration and are associated with the appearance of superconformal sectors in the F-theory model [10, 41-43].

One aspect of the connection between F-theory geometry and the anomaly structure of the associated $6 \mathrm{D}$ supergravity theory that we will find useful is the connection between the anomaly coefficient $b$ and the genus of the associated complex curve $C$ in the base $B$. We define a "genus" $g$ through

$$
2 g-2=b \cdot b+a \cdot b
$$

for any gauge factor with anomaly coefficient $b$ in a $6 \mathrm{D}$ supergravity theory. In the F-theory picture this is just the genus of the corresponding complex curve $C$; this genus formula can

\footnotetext{
${ }^{7}$ Because one chosen section $\sigma_{\infty}$, the "zero-section", serves as the additive identity for the group law on the elliptic fibers and for the Mordell-Weil group itself, the map $\sigma \mapsto[\sigma]$ which sends a rational section to its divisor class in $H^{1,1}(\tilde{X})$ is not a group homorphism. However, the modified map $\sigma \mapsto\left[\sigma-\sigma_{\infty}\right]$ does define a group homomorphism.
} 
be decomposed into contributions from each distinct matter representation, where some more exotic matter representations are associated with singularities in $C$ contributing to the arithmetic genus of $C[36,69]$.

The connection between F-theory geometry and physics for 4D F-theory constructions of supergravity theories is similar but has additional subtleties. The gauge group of the $4 \mathrm{D}$ theory again has a piece arising from 7-branes associated with Kodaira singularities in the elliptic fibration over codimension one loci (complex surfaces in this case) in the base threefold $B$, but additional gauge factors can arise from D3-branes in the type IIB picture. Furthermore, fluxes can break the geometric gauge group down to a smaller group, and the presence of a superpotential can push the complex structure moduli of the compactification to regions in moduli space with gauge enhancement. For further details concerning the structure of $4 \mathrm{D}$ theories that arise from F-theory see [44-48, 70].

\section{The completeness hypothesis and massless charge sufficiency}

\subsection{Statement of conditions}

The charge completeness hypothesis specialized to 4D and 6D supergravity states that

Charge completeness hypothesis for $4 \mathrm{D}$ and $6 \mathrm{D}$ supergravity. Consider any consistent $4 D \mathcal{N}=1$ or $6 D \mathcal{N}=(1,0)$ supergravity theory. States exist with all possible values in the charge lattice of the gauge group of the theory.

We state the condition of $6 \mathrm{D}$ massless charge sufficiency as follows:

Massless charge sufficiency condition in six dimensions. Consider any $6 D$ supergravity theory. The massless states in the theory with nontrivial charges under the gauge group generate a charge lattice $\Lambda$. We say that the theory satisfies the "massless charge sufficiency" condition when $\Lambda$ is the full charge lattice of the theory as given by the charge completeness hypothesis. Equivalently, the massless charge sufficiency condition states that the global structure of the gauge group is such that the group acts effectively on $\Lambda$, i.e., there is no element of the gauge group that acts trivially on all massless charged states.

\subsection{Proof of charge completeness hypothesis in F-theory}

We start with the completeness hypothesis in $6 \mathrm{D}$. The charge completeness hypothesis for the connected $\mathrm{U}(1)^{k}$ group of a 5D M-theory compactification on a smooth Calabi-Yau threefold $Y$ follows immediately from the fact that Poincaré duality gives a dual pairing between the geometric structures encoding the gauge bosons and the charged particles [28]. In this situation the $\mathrm{U}(1)$ gauge bosons $A_{i}$ arise from the M-theory 3 -form $C=A_{i} \wedge \omega_{i}$ where $\omega_{i}$ is a class in $H^{1,1}(Y, \mathbb{Z})$. States that are charged under the $\mathrm{U}(1)$ factors come from M2-branes wrapped on Poincaré dual 2-cycles in $H_{1,1}(Y, \mathbb{Z})$. The story in the corresponding F-theory is conceptually similar but slightly more subtle since some of the divisors in the corresponding Calabi-Yau threefolds are not associated with $6 \mathrm{D}$ gauge bosons and must be projected out to make a precise statement. 
Assume that a 6D supergravity theory has gauge group $G$ and comes from an F-theory Weierstrass model that defines an elliptic Calabi-Yau threefold $X$ with resolution $\tilde{X}$. We prove a "connected" version of the completeness hypothesis in which we only consider charges under the connected component of the identity in the gauge group - that is, we ignore discrete charges (if any). Our goal is to prove that the completeness hypothesis for a $6 \mathrm{D}$ supergravity theory realized through F-theory is equivalent to the condition that the global structure of the gauge group is identified through the standard assumption that the connected part of the gauge group is $G^{0}=\left(G_{0} \times \mathrm{U}(1)^{r}\right) / \Xi$, where $G_{0}$ is simply connected and $\pi_{1}\left(G^{0}\right)$ coincides with the Mordell-Weil group of the elliptic fibration. As discussed in section 2.2, each gauge boson in the Cartan subalgebra of $G$ is associated with a divisor $D \in H^{1,1}(\tilde{X})$. In the M-theory picture, these gauge bosons arise by reducing the M-theory three-form on the given divisor. ${ }^{8}$ All massive or massless charged states are associated (in the M-theory picture) with M2-branes wrapped on elements of $H_{2}(\tilde{X})$. By Poincaré duality there is a complex curve $C$ in $H_{1,1}(\tilde{X})$ with $D \cdot C=1$ for each divisor $D \in H^{1,1}(\tilde{X})$. More generally, there is a dual pairing between the linear space of divisors $D$ (with coefficients in $\mathbb{Z}$ ) and the space of complex curves $C$. Not all divisors in $H^{1,1}(\tilde{X})$ correspond to massless gauge bosons in the 6D F-theory model, however. From the Shioda-Tate-Wazir formula [72-74], we know that $h^{1,1}(\tilde{X})=\operatorname{rk} G+h^{1,1}(B)+1$; the divisors not corresponding to $6 \mathrm{D}$ gauge bosons are associated with the zero section $\sigma_{\infty}$ (which defines the additive identity in each elliptic fiber) and the pullbacks $\pi^{*} C_{\alpha}$ of divisors in the base, where $\left\{C_{\alpha}\right\}$ is a basis of divisors in the base. Thus, to apply Poincaré duality to get a dual pairing between gauge bosons and matter curves, we must separate out these extra divisors. The intersection of the zero section with the curve associated with the generic fiber $F$ is always $\sigma_{\infty} \cdot F=1$. Thus, we can always project away from this divisor-curve pair, considering only curves orthogonal to $\sigma_{\infty}$ and divisors orthogonal to $F$. The pullbacks $\pi^{*} C_{\alpha}$ are always orthogonal to $F, \pi^{*} C_{\alpha} \cdot F=0$. We can find a set of dual curves $\hat{C}_{\alpha}=\pi^{*} C_{\alpha} \cdot \sigma_{\infty}-\left(K_{B} \cdot C_{\alpha}\right) F$ that satisfy $\sigma_{\infty} \cdot \hat{C}_{\alpha}=0, \pi^{*} C_{\alpha} \cdot \hat{C}_{\beta}=\Omega_{\alpha \beta}$, where $\Omega_{\alpha \beta}$ is the intersection form on the base $B$, by projecting out the appropriate multiplicity of $F$, using the fact that, for any complex curve $C$ on the base, $\sigma_{\infty} \cdot \sigma_{\infty} \cdot \pi^{*} C=K_{B} \cdot C$ where $K_{B}$ is the canonical class of the base.

Projecting out these divisor-curve dual pairs, we are left with a dual pairing between the remaining divisors and curves. (Note that since $\Omega$ is unimodular for any surface, the divisors and curves that are pulled back from $B$ can always be projected out leaving a dual pairing between the remaining divisors and curves.) The remaining divisors consist of projections of sections (corresponding to the Mordell-Weil group), and projections of the Cartan divisors of the Lie algebra $\mathfrak{g}$. The projected sections take the form $\sigma-\sigma_{\infty}-\pi^{*} K_{B}$, where $\sigma-\sigma_{\infty}$ fill out the Mordell-Weil group $\mathbb{Z}^{r} \times T$ where $T$ is the Mordell-Weil torsion. The Cartan divisors of the Lie algebra are associated with the exceptional components of the Kodaira fibers of the codimension one singularities in the resolved fibration. Together, the Cartan divisors and the sections in the Mordell-Weil group (including the torsion elements) thus give a lattice which is dual to the charge lattice of the theory. Charge completeness holds precisely when these divisors generate the fundamental group of the

\footnotetext{
${ }^{8}$ Note that there are also non-Cartan gauge bosons, which are obtained in the M-theory picture as part of the spectrum determined by wrapping M2-branes on these same divisors [71].
} 
Cartan torus of the gauge group, where the curves lie in the dual lattice and therefore give all weights in the dual charge lattice $\Lambda$. This establishes the assertion that the (connected) completeness hypothesis for this class of theories is equivalent to the standard assumption that the fundamental group $\pi_{1}\left(G^{0}\right)$ of the connected component $G^{0}$ of the 6 D gauge group $G$ is generated by the Cartan divisors and the full Mordell-Weil group of sections of the CalabiYau threefold $X$. In particular, the global form of the gauge group is $G^{0}=\left(G_{0} \times \mathrm{U}(1)^{r}\right) / \Xi$, where $G_{0}$ is simply connected and $\Xi \cap G_{0}=T$ is the Mordell-Weil torsion. (See the appendix for more details about this group.)

Essentially the same argument goes through unchanged for $4 \mathrm{D} \mathcal{N}=1$ supergravity theories that come from F-theory on elliptic Calabi-Yau fourfolds, for the part of the gauge group that comes from Kodaira singularities over divisors in the threefold base and from sections. In $4 \mathrm{D}$ F-theory models, gauge bosons in this part of the gauge group are associated with divisors in the same way as in 6D theories. There are again matter states associated with M2-branes wrapped on complex curves in a similar way; note, however, that in the $4 \mathrm{D}$ construction these curves are fibral curves contained in a "matter surface" that lies over a codimension two locus in the base. Note also that, unlike in $6 \mathrm{D}$, in $4 \mathrm{D}$ F-theory constructions other connected gauge group factors can arise from, e.g., stacks of D3-branes, so the proof does not necessarily extend to the full connected gauge group. Note also that fluxes and the superpotential do not change this conclusion. While the superpotential can drive the theory to a locus with a larger geometric gauge group, the dual pairing between divisors and matter curves holds in the same fashion. And while fluxes can break the gauge group down from that naively determined by the 7-branes and Kodaira singularity structure, this simply removes part of the gauge group; the dual pairing between the remaining gauge components and matter charges must still hold.

\subsection{Massless charge sufficiency in 6D}

We conjecture that the massless charge sufficiency condition holds for the lattice of charges in each F-theory compactification giving a 6D supergravity theory, for all the (nonabelian or abelian) gauge factors (in the connected part of the gauge group) where the associated Green-Schwarz coefficient $b$ (or $\tilde{b}$ for abelian factors) satisfies the condition

$$
-a \cdot b>0
$$

where the inner product denotes the Dirac product on the string charge lattice. In F-theory this is equivalent to the condition that each such gauge factor is associated with a divisor $C$ satisfying $-K \cdot C>0$, where $K$ is the canonical class of the base $B$ and the inner product is the intersection form in the homology lattice of the compactification $\operatorname{space}^{9} H_{1,1}(B, \mathbb{Z})$.

There are two specific classes of cases where the positivity conditions (3.1) are violated and we are aware of theories that violate the massless charge sufficiency condition:

\footnotetext{
${ }^{9}$ As mentioned in the previous section, for nonabelian factors the divisor $C$ is the locus where the sevenbranes supporting the gauge factor are localized; for $\mathrm{U}(1)$ factors the divisor is somewhat more obscure but in the simplest cases can be associated with the divisor supporting an unHiggsed nonabelian factor containing the $\mathrm{U}(1)$.
} 
1) Cases where

$$
b \cdot b \leq-3 \text { and }-a \cdot b=b \cdot b+2 \leq-1 .
$$

These correspond to situations where the negative intersection $-K \cdot C<0$ gives rise to a "non-Higgsable" gauge factor [75], with minimal matter content (or possibly none at all), since the Weierstrass coefficients $f, g$ are forced to vanish along the geometric locus $C$ associated with $b$.

2) Cases where

$$
b \cdot b=-a \cdot b=0 .
$$

These cases are associated with gauge factors for which the matter content contains precisely one hypermultiplet in the adjoint representation and no other charged matter, as would be seen in a multiplet with enhanced supersymmetry.

We explore these two classes of exceptions further in section 6 . In fact, our conjecture is equivalent to the statement that massless charge sufficiency holds for all factors except those that fit into these two exceptional classes. This can be seen as follows: if the curve $C$ in the base associated with the anomaly coefficient $b$ is irreducible and $-K \cdot C<0$ it follows that ${ }^{10} C \cdot C=b \cdot b<0$. Since the genus $g$ in (2.11) is non-negative, we can only have a gauge factor associated with an irreducible curve $C$ and corresponding anomaly coefficient $b$ with $-a \cdot b<0$ when $g=0$ so $-a \cdot b=b \cdot b+2$ as in case (1). If $K \cdot C=0$ then the genus $g=1$ curve with $C \cdot C=0$ corresponds to the situation of case (2) giving our other class of exceptions. When $K \cdot C=0$ and the genus is higher, $g>1$, then $-a \cdot b=0, b \cdot b>0$, or $g=0$ so $b \cdot b=-2$, and it seems that massless charge sufficiency always holds, as we see in a number of cases in the following analysis.

While we do not have a mathematical proof of this conjecture, it holds in all cases we have analyzed, as we show in the following sections explicitly for various single-factor gauge groups. Here, we elaborate slightly further on the statement of this conjecture.

As described above, the connected part $G^{0}$ of the F-theory gauge group $G$ is generally a compact group of the form $G^{0}=\left(G_{0} \times \mathrm{U}(1)^{r}\right) / \Xi$, where $G_{0}$ is simply connected and $\Xi$ is a discrete subgroup of the center of $G_{0} \times \mathrm{U}(1)^{r}$. We can consider the charge lattice $\Lambda_{0}$ of the group $G_{0} \times \mathrm{U}(1)^{r}$. In any F-theory model, the massless states are associated with holomorphic or anti-holomorphic fibral curves (i.e. curves that project to a point in the base). The associated massless hypermultiplet states generate a sublattice $\Lambda \subset \Lambda_{0}$. Unless there are U(1) factors under which no fields are charged ("non-Higgsable U(1) factors" [7880]), the rank of $\Lambda$ is the same as the rank of $\Lambda_{0}$. Projecting out the charge lattice associated with such $\mathrm{U}(1)$ factors and with nonabelian factors having anomaly coefficients that violate (3.1), to give the lattice $p\left(\Lambda_{0}\right)$, and similarly projecting out the charges under nonabelian factors violating (3.1) from $\Lambda$, the quotient $\Xi^{\prime}=p\left(\Lambda_{0}\right) / p(\Lambda)$ is the subgroup of the center of $G_{0}$ that leaves all the remaining massless charged states invariant. Our conjecture is then that $\Xi=\Xi^{\prime}$.

\footnotetext{
${ }^{10}$ This can be shown for example by noting that such a negative intersection product is only possible for irreducible $C$ when $-K$ contains $C$ as a component; it follows that we can write $-K=n C+R$ with $R \cdot C \geq 0$ (Zariski decomposition [76, 77]), so $C \cdot C<0$.
} 
In the next section we analyze the possible Mordell-Weil torsion groups $T$ for nonabelian gauge groups. We show that for many of the gauge factors that contain each such group $\Xi=T$ in the center, when the anomaly coefficient satisfies (3.1) it follows that massless charge sufficiency always holds. In particular, under these circumstances all anomaly-consistent massless spectra that are invariant under $\Xi=T$ are associated with an F-theory Weierstrass model that indeed has this torsion group, so that $\Xi=\Xi^{\prime}$ in all of these cases. In section 5 we give an example of massless charge sufficiency for an abelian $\mathrm{U}(1)$ theory, and in section 6 , we describe in more detail the cases that violate (3.1) and the massless charge sufficiency condition

\section{Examples: massless charge sufficiency and Mordell-Weil torsion for nonabelian gauge groups}

We present here a variety of examples of $6 \mathrm{D}$ supergravity theories with purely nonabelian gauge groups that are anomaly free and have a massless spectrum that is invariant under a nontrivial discrete subgroup of the gauge group. These are the situations in which the massless charge sufficiency condition has the potential to fail. We show that in all these cases except those where the associated anomaly coefficient $b$ satisfies (3.2) or (3.3) the Mordell-Weil group of any F-theory construction with the desired gauge algebra and matter content has the appropriate torsion subgroup $T=\Xi$, where $G^{0}=G_{0} / \Xi$, so that the massless charge sufficiency condition is satisfied, in accord with our conjecture. We focus primarily here on theories with "generic" matter representations [81], which for a single $\mathrm{SU}(N)$ factor, for example, consists only of the fundamental, adjoint and two-index antisymmetric representations. We explicitly analyze one case of exotic (non-generic) matter in section 4.2 (the 3-index antisymmetric representation of $\mathrm{SU}(6)$ ). The analysis of [69] suggests that there are few other viable exotic matter representations compatible with Mordell-Weil torsion; in particular, the 3-index (4) representation of SU(2) breaks the possible $\mathbb{Z}_{2}$ central subgroup, while the 2-index symmetric representation of a general $\mathrm{SU}(N)$ group can preserve the $\mathbb{Z}_{2}$ subgroup of $\mathbb{Z}_{N}$ for even $N$. In general, including more exotic matter types does not seem to modify the conclusions found here; we make a few comments about this in various appropriate places but do not make an effort towards a complete analysis of such questions. In this section and the following sections we use some basic aspects of the geometry of elliptic curves; for a good introduction to these topics and related methods see $[82,83]$.

We organize the analysis by the Mordell-Weil torsion group, and consider for each torsion group different gauge groups that may arise with that torsion subgroup. While our analysis here is not completely exhaustive, we give a fairly complete analysis of the possibilities for nonabelian gauge groups.

From the geometry of elliptic Calabi-Yau manifolds we know that the only possible torsion groups that can appear in the Mordell-Weil group for an elliptic Calabi-Yau threefold or fourfold are the following groups: ${ }^{11}$

$$
\mathbb{Z}_{m}, 2 \leq m \leq 6 ; \quad \mathbb{Z}_{2} \oplus \mathbb{Z}_{2 n}, 1 \leq n \leq 2 ; \quad \mathbb{Z}_{3} \oplus \mathbb{Z}_{3}
$$

\footnotetext{
${ }^{11}$ We use additive notation for these groups, which are always abelian.
} 


\begin{tabular}{|c|c|c|}
\hline gauge algebra & simply connected $G_{0}$ & center \\
\hline $\mathfrak{s u}(n)$ & $\mathrm{SU}(n)$ & $\mathbb{Z}_{n}$ \\
$\mathfrak{s p}(n)$ & $\operatorname{Sp}(n)$ & $\mathbb{Z}_{2}$ \\
$\mathfrak{s o}(4 n)$ & $\operatorname{spin}(4 n)$ & $\mathbb{Z}_{2} \oplus \mathbb{Z}_{2}$ \\
$\mathfrak{s o}(4 n+2)$ & $\operatorname{spin}(4 n+2)$ & $\mathbb{Z}_{4}$ \\
$\mathfrak{s o}(2 n+1)$ & $\operatorname{spin}(2 n+1)$ & $\mathbb{Z}_{2}$ \\
$\mathfrak{e}_{6}$ & $E_{6}$ & $\mathbb{Z}_{3}$ \\
$\mathfrak{e}_{7}$ & $E_{7}$ & $\mathbb{Z}_{2}$ \\
$\mathfrak{e}_{8}$ & $E_{8}$ & 1 \\
$\mathfrak{f}_{4}$ & $F_{4}$ & 1 \\
$\mathfrak{g}_{2}$ & $G_{2}$ & 1 \\
\hline
\end{tabular}

Table 1. The discrete central subgroup of the simply connected groups associated with each gauge algebra.

Explicit forms of the Weierstrass models associated with each of these discrete groups were given in [27], following [84]. It was argued in [85] that these are the only possible torsion groups for elliptic Calabi-Yau manifolds. ${ }^{12}$ We consider in turn the Weierstrass forms and 6D supergravity models associated with theories with the torsion groups (4.1).

On the $6 \mathrm{D}$ supergravity side, we consider models with different nonabelian gauge groups. Abelian gauge factors are more subtle and we address some questions about U(1) factors in the following section. For nonabelian gauge algebras that can appear in 6D supergravity theories, the discrete central subgroup of the simply connected associated gauge group is listed in table 1 , following [88].

\section{1 $\mathbb{Z}_{2}$ torsion}

\subsubsection{General Weierstrass models with $\mathbb{Z}_{2}$ torsion}

A Weierstrass model that has a section of order 2 , corresponding to a $\mathbb{Z}_{2}$ factor in the Mordell-Weil group, must have the form [27]

$$
y^{2}=x\left(x^{2}+\alpha_{2} x+\alpha_{4}\right) .
$$

This can be seen simply from the elliptic curve addition law, which states that for a given elliptic curve $C$ the three points $p, q, r$ that lie on the intersection $L \cdot C$ with any line $L$ satisfy $p+q+r=0$, and the condition that the zero point $\mathcal{O}$ of the elliptic curve lies at the point at infinity along any vertical line $x=$ const in the $x, y$ plane. From these conditions, we see that a point $p$ satisfies $2 p=p+p=0$ if and only if the tangent to the cubic at $p$ is vertical, so shifting the point $p$ to the origin gives the Weierstrass form (4.2).

Completing the square to get the reduced form we have

$$
y^{2}=x^{3}+f x+g
$$

\footnotetext{
${ }^{12}$ Note that, as shown by Mazur $[86,87]$, other groups such as $\mathbb{Z}_{7}, \ldots, \mathbb{Z}_{10}, \mathbb{Z}_{12}$ and $\mathbb{Z}_{2} \oplus \mathbb{Z}_{6}, \mathbb{Z}_{2} \oplus \mathbb{Z}_{8}$ are possible for an elliptic curve over $\mathbb{Q}$, though $\mathbb{Z}_{3} \oplus \mathbb{Z}_{3}$ is not allowed over $\mathbb{Q}$.
} 
where

$$
\begin{aligned}
& f=-\alpha_{2}^{2} / 3+\alpha_{4} \\
& g=2 \alpha_{2}^{3} / 27-\alpha_{2} \alpha_{4} / 3 .
\end{aligned}
$$

The discriminant then has a factor

$$
\Delta=4 f^{3}+27 g^{2}=\alpha_{4}^{2} \tilde{\Delta},
$$

implying at least a gauge algebra $\mathfrak{s u}(2)$ on the locus $\alpha_{4}=0$.

\subsection{2 $\mathfrak{s u ( 2 )}$ Weierstrass models with $\mathbb{Z}_{2}$ torsion}

We begin with models with a gauge algebra $\mathfrak{s u}(2)=\mathfrak{s p}(1)$. The generic form of Weierstrass model giving a gauge algebra $\mathfrak{s u}(2)$ (which generically gives an $\mathrm{SU}(2)$ group that has only fundamental and adjoint matter) on a curve $\sigma=0$ can be constructed from the Tate form [66, 89], and by direct analysis of the general Weierstrass model with the appropriate Kodaira singularity type [68]; from the latter perspective it is clear that this Weierstrass model is the most general of this type and has all the moduli associated with this branch of $6 \mathrm{D}$ supergravity theories. Such a generic SU(2) Weierstrass model has

$$
\begin{aligned}
& f=-\frac{1}{48} \phi^{2}+\sigma f_{1} \\
& g=\frac{1}{864} \phi^{3}-\frac{1}{12} \phi \sigma f_{1}+\sigma^{2}\left(g_{2}\right),
\end{aligned}
$$

which can be related to the Tate parameters $a_{k}=\sigma^{n_{k}} \tilde{a}_{k}$ (with $n_{1}, n_{2}, n_{3}, n_{4}, n_{6}=0,0,1$, $1,2)$ by

$$
f=-\frac{1}{48}\left(a_{1}^{2}+4 a_{2}\right)^{2}+\left(\tilde{a}_{4}+a_{1} \tilde{a}_{3} / 2\right) \sigma
$$

and

$$
g=\frac{1}{864}\left(a_{1}^{2}+4 a_{2}\right)^{3}-\frac{1}{12}\left(a_{1}^{2}+4 a_{2}\right)\left(\tilde{a}_{4}+a_{1} \tilde{a}_{3} / 2\right) \sigma+\left(\tilde{a}_{6}+\tilde{a}_{3}^{2} / 4\right) \sigma^{2} .
$$

We thus identify $\phi=a_{1}^{2}+4 a_{2}, f_{1}=\tilde{a}_{4}+a_{1} \tilde{a}_{3} / 2, g_{2}=\tilde{a}_{6}+\tilde{a}_{3}^{2} / 4$, and we see that the Tate formulation has a redundancy under the choice of $a_{1}, a_{3}$. Matter in the fundamental representation of $\mathfrak{s u}(2)$ transforms nontrivially under the $\mathbb{Z}_{2}$ center, while matter in the adjoint representation does not, so the possibility of having a group of global structure $\mathrm{SO}(3)=\mathrm{SU}(2) / \mathbb{Z}_{2}$ can only arise in the absence of matter in the fundamental representation.

The discriminant locus of (4.6) takes the form

$$
\Delta=\frac{1}{16} \phi^{2}\left(\phi g_{2}-f_{1}^{2}\right) \sigma^{2}+\mathcal{O}\left(\sigma^{3}\right) .
$$

Fundamental matter fields arise at the vanishing locus of the factor $\left(\phi g_{2}-f_{1}^{2}\right)$. In general, $\phi$ is a section of $\mathcal{O}(-2 K)$, and $\Delta$ is a section of $\mathcal{O}(-12 K)$.

It is straightforward to confirm that when $g_{2}=0$, the Weierstrass model (4.6) takes the form (4.4) and has $\mathbb{Z}_{2}$ torsion. We now prove that this is the case whenever $-a \cdot b>$ 
0 and there is no fundamental matter, only matter in the adjoint of $\mathfrak{s u}(2)$. From the anomaly cancellation conditions it is straightforward to determine that the number of hypermultiplet fields in the fundamental representation of a theory with $\mathfrak{s u}(2)$ algebra is $-8 a \cdot b-2 b \cdot b$ (assuming as stated above that we have only generic matter types, so that the only representation arising is adjoint matter). To have no fundamental matter fields, we must then have the condition

$$
(-4 a-b) \cdot b=0
$$

If $-a \cdot b>0$, we must then also have $b \cdot b=-4 a \cdot b>0$. The class of $g_{2}$ is $\left[g_{2}\right]=-6 a-2 b$. It thus follows that $\left[g_{2}\right] \cdot b=-b \cdot b / 2<0$. This, however, implies that $\left[g_{2}\right]$ is not an effective class, since if $b$ is an irreducible effective class with $b \cdot b>0$ there is no effective class $c$ with $c \cdot b<0$. This shows that $g_{2}$ must vanish, so the absence of fundamental matter implies the presence of the Mordell-Weil torsion section associated with the $\mathbb{Z}_{2}$ quotient of the gauge group. This demonstrates that, at least for theories with only the generic fundamental and adjoint matter representations of a gauge factor $\mathfrak{s u}(2)$, when the condition $-a \cdot b>0$ is satisfied the massless charge sufficiency condition holds. Note that for the gauge algebra $\mathfrak{s u}(2)$, the only exceptions to massless charge sufficiency are those of class (2) where the condition (3.3) holds. When $b \cdot b \leq-3$, the rank of the resulting non-Higgsable gauge algebra is at least two. And when $-a \cdot b=0, b \cdot b=-2$, we have fields in the fundamental matter representation so the charge lattice is automatically filled.

For the gauge algebra $\mathfrak{s u}(2)$, there cannot be any further exceptions to massless charge sufficiency even when other matter representations that can be realized through F-theory geometry are included. It is argued in [69] that the only exotic matter representation that can arise in F-theory for $\mathfrak{s u}(2)$ is the 3 -index (4) representation. Since this representation by itself is sufficient to generate the full $\mathrm{SU}(2)$ charge lattice, any model that includes this representation automatically satisfies massless charge sufficiency.

It is interesting to consider explicitly some of the classes of cases with generic matter where the massless charge sufficiency condition holds. When $\sigma$ is in the class $-4 K, f_{1}$ becomes a constant and $g_{2}$ automatically vanishes. In this case, as discussed above, there is no (massless) matter charged in the fundamental representation of the $\mathfrak{s u}(2)$, only adjoint matter. The presence of the $\mathbb{Z}_{2}$ torsion in this case indicates that the global form of the gauge group is $\mathrm{SO}(3)=\mathrm{SU}(2) / \mathbb{Z}_{2}$, in agreement with the massless charge sufficiency hypothesis.

A special case of the $\mathrm{SU}(2) / \mathbb{Z}_{2}$ Weierstrass model occurs when $\phi=0$ and $[\sigma]=-4 K_{B}$. In this case, $g$ vanishes identically and we have a Kodaira type III singularity, giving an alternative realization of the $\mathfrak{s u}(2)$ algebra.

When $\sigma$ is in a class such that $\left[g_{2}\right]=-6 K_{B}-2[\sigma]$ is ineffective (there are no sections of the corresponding line bundle), then $g_{2}$ automatically vanishes. In such a case, as long as $f_{1}$ is in an effective class (has sections), we get another $\mathrm{SU}(2)$ factor on the locus $f_{1}=0$ (or several SU(2) factors if $f_{1}$ is reducible). ${ }^{13}$ This can also occur when $g_{2}$ is in an effective

\footnotetext{
${ }^{13}$ This is a simple example of the automatic enhancement conjecture described in [19], and was part of the motivation for the work in that paper.
} 
class but tuned to vanish. In either of these cases, we again have $\mathbb{Z}_{2}$ torsion, but the global form of the gauge group is $G=(\mathrm{SU}(2) \times \mathrm{SU}(2)) / \mathbb{Z}_{2}$ (with more factors if $f_{1}$ or $\sigma$ is reducible). (Note that if $f_{1}$ is not in an effective class, or otherwise vanishes along with $g_{2}$, then $f, g$ vanish to order $(2,3)$ at the vanishing locus of $\phi$, which supports a larger gauge group such as $\mathrm{SO}(8)$.)

As simple examples of these situations, consider the base $\mathbb{P}^{2}$, with canonical class $-K_{B}=3 H$. When $\sigma$ is a degree 12 curve, we have an $\mathrm{SO}(3)$ theory with only adjoint matter (55 adjoints). When $[\sigma]<12$ and $g_{2}=0$ we have an $(\mathrm{SU}(2) \times \mathrm{SU}(2)) / \mathbb{Z}_{2}$ theory where the divisors supporting the two $\mathrm{SU}(2)$ factors have degrees $d, 12-d$. When the degree of $\sigma$ is 10 or 11 , this condition is forced automatically, so there is no pure $\mathrm{SU}(2)$ theory with anomaly coefficient $b=d=10,11$ that comes from such an F-theory model at $T=0$. Such theories are anomaly-consistent, however, so form a part of the apparent $6 \mathrm{D}$ supergravity "swampland".

Note that the cases with gauge group $(\mathrm{SU}(2) \times \mathrm{SU}(2)) / \mathbb{Z}_{2}$ can be thought of as degenerate cases of the $\mathrm{SO}(3)$ theory where the divisor supporting the gauge group becomes reducible. In particular, when $[\sigma]+\left[f_{1}\right]=-4 K$, this can be thought of as tuning an $\mathrm{SO}(3)$ on a reducible divisor $\alpha_{4}=\sigma f_{1}$.

From the $6 \mathrm{D}$ supergravity point of view, the models described correspond precisely with the set of anomaly-consistent models with only $\mathrm{SU}(2)$ gauge group factors and generic matter content that is invariant under a central $\mathbb{Z}_{2}$. For example, at $T=0$ the number of fundamental matter representations of an $\mathrm{SU}(2)$ with anomaly coefficient $b>0$ is easily computed to be $2 b(12-b)$. This vanishes only when $b=12$, corresponding to the $\mathrm{SO}(3)$ theory described above, and the fundamentals are all associated with bifundamentals with other $\mathrm{SU}(2)$ factors precisely when the anomaly coefficients of the $\mathrm{SU}(2)$ factors satisfy $\sum_{i} b_{i}=12$.

\subsection{3 $\quad \mathbb{Z}_{2}$ torsion and larger $\mathrm{SU}(N)$ and $\operatorname{Sp}(N)$ groups}

For groups $\mathrm{SU}(2 N)$ with $N>1$, the discrete $\mathbb{Z}_{2}$ subgroup of the center $\mathbb{Z}_{2 N}$ is respected by models that have no fundamental matter but do have matter in the 2-index antisymmetric and adjoint representations. The $\mathbb{Z}_{2}$ center of $\operatorname{Sp}(N)$ models without fundamental matter is similarly preserved, and the anomaly equation for the number of fundamental matter fields is the same in these cases. A variety of such models with larger $\mathrm{SU}(N)$ and $\operatorname{Sp}(N)$ groups can be constructed by taking $\alpha_{4}=\sigma^{k}$ in (4.4) for some integer $k$. In this case the gauge group on the locus $\sigma=0$ becomes $\mathrm{SU}(2 k)$ when $\alpha_{2}$ is a perfect square, and otherwise is $\operatorname{Sp}(k)$. Since $\alpha_{4}$ is in the class $-4 K$, the divisor $4 K$ must be divisible by $k$ in $\operatorname{Pic}(B)$ in order to carry out such a construction. For example, on the base $\mathbb{P}^{2}$ we have such a model for any $k \mid 12$, so that there are $T=0$ models with gauge groups $\mathrm{SO}(3), \mathrm{SU}(4) / \mathbb{Z}_{2}, \mathrm{SU}(6) / \mathbb{Z}_{2}, \mathrm{SU}(8) / \mathbb{Z}_{2}, \mathrm{SU}(12) / \mathbb{Z}_{2}$, and $\mathrm{SU}(24) / \mathbb{Z}_{2}$ (and similarly for the analogous $\operatorname{Sp}(N) / \mathbb{Z}_{2}$ models). In each case there are no fundamental matter fields, only adjoint and two-index antisymmetric tensor fields. The Weierstrass model for $\mathrm{SU}(24) / \mathbb{Z}_{2}$ was considered previously in [68] (section 4.1). It is not hard to check that these are the only anomaly-free models with $T=0$ and gauge algebra $\mathfrak{s u}(N)$ or $\mathfrak{s p}(N)$ with generic matter representations and no matter in the fundamental representation. Note that there 
may be related models (in some cases connected by "matter transitions" [90]) in which there are two-index symmetric and antisymmetric matter representations replacing adjoints [69], which could still preserve the $\mathbb{Z}_{2}$ subgroup; we leave further analysis of such possible exotic matter models as open problems. Other models with a variety of product gauge groups can be constructed by making $\alpha_{4}$ reducible. For example, taking $T=0$ and $\alpha_{4}=\phi_{4}^{2} \phi_{2}^{2}$ gives a theory with gauge group $(\mathrm{SU}(4) \times \mathrm{SU}(4)) / \mathbb{Z}_{2}$ on degree 4 and 2 curves in $\mathbb{P}^{2}$, so that the matter content consists of 3 adjoint representations of the first SU(4), 8 bifundamental matter fields, and 12 and 6 two-index antisymmetric matter fields for the two SU(4) factors respectively.

Note that making $\alpha_{4}$ reducible in the special case where $\alpha_{2}=0$ enhances the Kodaira type III singularity mentioned above to a type $I_{0}^{*}(\mathrm{SO}(8))$ or type $\mathrm{III}^{*}\left(E_{7}\right)$ singularity when $\alpha_{4}=\sigma^{2}$ or $\alpha_{4}=\sigma^{3}$ respectively, as discussed further below.

Considering $\mathrm{SU}(N)$ models with $T>0$, a similar, though slightly more intricate, analysis to that of $\mathrm{SU}(2)$ allows us to prove that just as for $\mathrm{SU}(2)$, for any $\mathrm{SU}(4)$ model (for any $T$ ) where $-a \cdot b>0$ that has only generic matter invariant under the $\mathbb{Z}_{2}$ subgroup of the center the associated Weierstrass model has $\mathbb{Z}_{2}$ torsion. This can be seen as follows: from [68], the general form of a Weierstrass model with $\mathrm{SU}(4)$ symmetry on the divisor $\sigma$ is

$$
\begin{aligned}
& f=-\frac{1}{48} \phi_{0}^{4}-\frac{1}{6} \phi_{0}^{2} \phi_{1} \sigma+f_{2} \sigma^{2}+f_{3} \sigma^{3} \\
& g=\frac{1}{864} \phi_{0}^{6}+\frac{1}{72} \phi_{0}^{4} \phi_{1} \sigma+\left(\frac{1}{36} \phi_{0}^{2} \phi_{1}^{2}-\frac{1}{12} \phi_{0}^{2} f_{2}\right) \sigma^{2}+\left(-\frac{1}{12} \phi_{0}^{2} f_{3}-\frac{1}{3} \phi_{1} f_{2}-\frac{1}{27} \phi_{1}^{3}\right) \sigma^{3}+g_{4} \sigma^{4},
\end{aligned}
$$

where $f_{3}, g_{4}$ may also include terms of higher order in $\sigma$. From anomaly cancellation for a theory with only generic (adjoint, two-index antisymmetric, fundamental) matter, the number of fields in the fundamental representation is $-8 a \cdot b-4 b \cdot b$, so to have no matter fields we must have

$$
(-2 a-b) \cdot b=0 .
$$

If $-a \cdot b>0$, it follows that $b \cdot b>0$. The class of $f_{3}$ is $\left[f_{3}\right]=-4 a-3 b$, which must be ineffective. Similarly, $g_{4}$ is ineffective, so $f_{3}=g_{4}=0$. The remaining nonzero terms in the Weierstrass model (4.11) then take the form (4.4), where

$$
\begin{aligned}
\alpha_{2} & =\frac{1}{4} \phi_{0}^{2}+\phi_{1} \sigma \\
\alpha_{4} & =\left(f_{2}+\frac{1}{3} \phi_{1}^{2}\right) \sigma^{2} .
\end{aligned}
$$

This shows that our conjecture on massless charge sufficiency holds for SU(4) models with only two-index antisymmetric and adjoint matter. The only other representation possible in standard F-theory models is the two-index symmetric representation [69], and the adjoint is anomaly equivalent to a combination of the two-index symmetric and antisymmetric representations, so the condition for the number of fundamental representations to vanish is the same in the presence of this exotic matter. Thus, for $\mathrm{SU}(4)$ we have proven the massless charge sufficiency conjecture for arbitrary matter fields. The same argument holds for models with a gauge group $\mathrm{Sp}(2)$; the only difference is that we replace the 
perfect square $\phi_{0}^{2}$ (that satisfies the "split" condition) in the Weierstrass model with a more general field $\phi$.

A similar argument holds for $\mathrm{SU}(2 k)$ models with larger $N=2 k$. While for $\mathrm{SU}(6)$ and $\mathrm{SU}(8)$ the story is slightly more complicated due to the possible presence of 3-index antisymmetric matter representations, the most generic type of $\mathrm{SU}(2 k)$ tuning takes the form $[68]$

$$
\begin{aligned}
& f=-\frac{1}{3} \Phi^{2}+f_{k} \sigma^{k}+f_{k+1} \sigma^{k+1} \\
& g=-\frac{2}{27} \Phi^{3}-\frac{1}{3} \Phi\left(f_{k} \sigma^{k}+f_{k+1} \sigma^{k+1}\right)+g_{2 k} \sigma^{2 k} .
\end{aligned}
$$

The number of fundamental matter fields is given by $-8 a \cdot b-2 k b \cdot b$, so to have no fundamental matter fields we must have

$$
(-4 a-k b) \cdot b=0
$$

The classes of $f_{k+1}$ and $g_{2 k}$ are $-4 a-(k+1) b$ and $-6 a-2 k$, which are both ineffective through similar arguments to the above, so these terms vanish and the absence of fundamental matter with $-a \cdot b$ thus implies that we have the $\mathbb{Z}_{2}$ torsion form (4.4). Again, the analysis is parallel for $\operatorname{Sp}(k)$. All these models thus agree with the conjecture for massless charge sufficiency.

\subsection{4 $\quad E_{7}$ gauge group}

The group $E_{7}$ has a center $\mathbb{Z}_{2}$. The generic matter representations are the adjoint $(\mathbf{1 3 3})$ and fundamental (56) representations. No higher-dimensional exotic $E_{7}$ representations should be possible in standard F-theory models. The adjoint is invariant under the $\mathbb{Z}_{2}$ center, while the fundamental is not. Thus, the only situation where we can have a matter spectrum with generic matter that respects the $\mathbb{Z}_{2}$ symmetry is when we only have adjoint matter. We get an $\mathfrak{e}_{7}$ algebra over a divisor $\sigma=0$ for a Weierstrass model with $f, g$ of the form

$$
\begin{aligned}
f & =\sigma^{3} \tilde{f} \\
g & =\sigma^{5} \tilde{g} \\
\Delta & =4 \sigma^{9} \tilde{f}^{3} .
\end{aligned}
$$

Fundamental matter appears at the locus where $\tilde{f}=0$.

We now prove that the massless charge sufficiency conjecture holds by demonstrating that the absence of fundamental matter and the condition $-a \cdot b$ together imply the presence of $\mathbb{Z}_{2}$ torsion. The number of (half-hypermultiplet) fundamental matter fields is given by $(-4 a-3 b) \cdot b$. When this quantity vanishes, it follows that $\tilde{g}$, with class $[\tilde{g}]=-6 a-5 b$ has an ineffective class and vanishes. We thus match the Weierstrass form (4.2) with $\alpha_{2}=0, \alpha_{4}=\tilde{f} \sigma^{3}$ when the $E_{7}$ theory has only adjoint matter, giving another class of examples where the massless charge sufficiency conjecture holds. 
In the simplest cases with only adjoint matter $\tilde{f}$ is simply a constant, and $\sigma$ is a section of $-(4 / 3) K_{B}$. This is possible, for example, on the base $\mathbb{P}^{2}$ when $\sigma$ is a quartic polynomial. Other models with $\mathbb{Z}_{2}$ torsion can arise when $T>0$.

One other relevant class of models occurs when $\tilde{g}=0$ but $\tilde{f}$ is not a constant. This corresponds to the case discussed in the previous subsections where $\alpha_{2}=0$ and $\alpha_{4}$ is reducible, where we have a product group with both an $E_{7}$ and other factors.

\subsection{5 $\mathrm{SO}(N)$ and $\mathbb{Z}_{2}$ torsion}

A theory with an $\mathfrak{s o}(N)$ gauge algebra and generic matter generally has both fundamental matter and matter in the spinor representation, in addition to adjoint matter. We focus here first on the cases $\mathfrak{s o}(2 n+1)$, where the center of the simply connected group $\operatorname{spin}(2 n+1)$ is $\mathbb{Z}_{2}$.

The Weierstrass model for an $\mathfrak{s o}(2 n+7)$ or $\mathfrak{s o}(2 n+8)$ gauge algebra has the form

$$
\begin{aligned}
f & =f_{2} \sigma^{2}+f_{3} \sigma^{3} \\
g & =g_{3} \sigma^{3}+g_{4} \sigma^{4} \\
\Delta & =\Delta_{6+n} \sigma^{6+n}
\end{aligned}
$$

where the choice of $2 n+7$ or $2 n+8$ depends upon additional monodromy conditions, and the last coefficients in each expansion may contain higher order powers in $\sigma$.

The spin representation breaks the central $\mathbb{Z}_{2}$ for odd $N$, so we can have $\mathbb{Z}_{2}$ torsion with gauge algebra $\mathfrak{s o}(2 n+1)$ only when there are no fields in the spin representation. By anomalies this occurs only when $(-2 a-b) \cdot b=0$. In this circumstance, when $-a \cdot b>$ 0 it follows that $f_{3}, g_{4}$ vanish. The discriminant is then $\Delta=\left(4 f_{2}^{2}+27 g_{3}^{3}\right) \sigma^{6}$, so this is only possible for $\mathfrak{s o}(7)$ when $N$ is odd. The $\mathfrak{s o}(7)$ monodromy condition is that the cubic $x^{3}+f_{2} x+g_{3}$ factorizes as $(x-A)\left(x^{2}+A x+B\right)$, while a factorization of the form $(x-A)(x-B)(x+(A+B))$ gives $\mathfrak{s o}(8)$. When $(-2 a-b) \cdot b=0$ and $-a \cdot b>0$ we have $b \cdot b>0$, and it follows that $-2 a-b$ is a rigid divisor (or sum of rigid divisors). ${ }^{14}$ It then follows that $f_{2}=c A^{2}, g_{3}=c^{\prime} A^{3}$ with $c, c^{\prime}$ constant and $[A]=-2 a-b$. This implies that the monodromy is that of $\mathfrak{s o}(8)$. In this case in fact the torsion is $\mathbb{Z}_{2} \oplus \mathbb{Z}_{2}$, as we discuss further in section 4.3.2. This proves that the massless charge sufficiency conjecture we have made holds for gauge algebras $\mathfrak{s o}(2 n+1)$ taken in isolation. In fact, we have shown that there are no situations at all where a pure $\mathfrak{s o}(2 n+1)$ gauge algebra arises without some matter in the spinor representation when $-a \cdot b>0$.

We could also get a $\mathbb{Z}_{2}$ torsion by breaking part of the discrete center of the gauge group when the algebra is $\mathfrak{s o}(2 n)$ with $2 n$ even. For the algebra $\mathfrak{s o}(4 n+2)$, there are two spinor representations and either spin representation again completely breaks the central $\mathbb{Z}_{4}[88]$.

\footnotetext{
${ }^{14}$ We argue in favor of this by using the Zariski decomposition $[76,77]$ of the effective $\mathbb{Q}$-divisor $-2 a$. (This divisor is effective because $-2 a=-2 K_{B}$ and the Weierstrass coefficients $f$ and $g$ are sections of $-4 K_{B}$ and $-6 K_{B}$, respectively.) We assume that the gauge divisor $C$ is irreducible; since $C \cdot C=b \cdot b>0$, it follows that $b$ is nef (since its intersection with any irreducible divisor other than $C$ must be nonnegative). Thus, $-2 a=b+(-2 a-b)$ must be the Zariski decomposition of $-2 a$, so that in particular, if we write $-2 a-b$ in terms of irreducible components, the intersection matrix of those components must be negative definite. In particular, each component has negative self-intersection and so it is rigid.
} 
From the same argument as above, the absence of matter in a spinor representation implies $(-2 a-b) \cdot b=0$, and there are no models with $-a \cdot b>0$ and no spinors except $\mathfrak{s o}(8)$. For the gauge algebra $\mathfrak{s o}(N)$ with $N=4 n$, the presence of either of the spinor representations or the fundamental representation leaves a $\mathbb{Z}_{2}$ invariant, but the center is completely broken by any pair of these representations. When the genus $g$ defined through (2.11) is one, then the multiplicities of both spinor and fundamental representations are given by $b \cdot b$, so the center is completely broken except in the exceptional case $-a \cdot b=b \cdot b=0$. When $g=0$, the number of spinors is $b \cdot b+4$, which can only vanish when $b \cdot b=-4$, another exceptional case that we return to in section 6.1 , and the number of fundamental representations is $b \cdot b+N-4$, which is always larger than or equal to the number of spinors, so the only cases where the discrete center is not completely broken are exceptional. When $g>1$, on the other hand, there are always more spinors than fundamental representations when $N>8$; the case of $\mathfrak{s o}(8)$ is treated in section 4.3.2. This leaves as a possible class of exceptions to massless charge sufficiency theories with algebra $\mathfrak{s o}(4 n)$ and spinor matter but no fundamental matter. For example, on the base $\mathbb{P}^{2}$ we can tune a $\mathfrak{s o}(12)$ algebra on a quartic (genus $g=3$ ), and the matter content consists of 8 spinor fields and no fundamental fields. If all the spinors appearing here are in the same representation, this would violate massless charge sufficiency unless the corresponding Weierstrass model has $\mathbb{Z}_{2}$ Mordell-Weil torsion. We can check explicitly, however, that in such a case the Weierstrass model does have the form (4.4). For the number of fundamental fields to vanish for $\mathfrak{s o}(12)$, we have $(-4 a-3 b) \cdot b=0$. From this it follows that the Tate coefficients $a_{1}, a_{3}, a_{6}$ must vanish in the standard $\mathfrak{s o}(12)$ Tate tuning, giving precisely the resulting Weierstrass model form (4.4). It is not possible to have an $\mathfrak{s o}(16)$ or higher gauge algebra on a curve with $b \cdot b>0$, so we have checked massless charge sufficiency for all possible $\mathfrak{s o}(n)$ algebras with $\mathbb{Z}_{2}$ torsion.

Considering product groups associated with gauge algebras with multiple components, it would seem that various combinations of $\mathfrak{s u}(2), \mathfrak{s o}(7)$, and $\mathfrak{e}_{7}$ algebras can be produced with a single $\mathbb{Z}_{2}$ torsion factor by taking the class of $\mathbb{Z}_{2}$ models with $\alpha_{2}=0$ and setting $\alpha_{4}$ to be a product of various linear, quadratic, and cubic factors. The $\mathfrak{s o}(7)$ part of the Weierstrass model then takes the form of (4.18), with $f_{3}=g=0$. While taken alone the $\mathfrak{s o}(8)$ symmetry coming from the quadratic factors would respect a $\mathbb{Z}_{2} \oplus \mathbb{Z}_{2}$ torsion as described below, the monodromy condition is changed to give an $\mathfrak{s o}(7)$ and the discrete symmetry is broken to $\mathbb{Z}_{2}$ in the presence of at least one linear or cubic factor in $\alpha_{4}$. When the only gauge factors are $\mathrm{SU}(2)$ and a single $\mathrm{SO}(7)$, this gives a perfectly well-behaved set of models where all matter is in $(\mathbf{2}, \mathbf{8})$ representations at the intersections of the $\mathrm{SU}(2)$ divisors with the $\mathrm{SO}(7)$ divisor; this matter is invariant under the $\mathbb{Z}_{2}$ that acts simultaneously on all factors. Including more than one $\mathrm{SO}(7)$ or $E_{7}$ factor gives $(4,6)$ points at the intersections of the divisors associated with these factors (unless we are in the special case where $-a \cdot b=b \cdot b=0$ for these factors). An interesting set of examples of this type with at least two $E_{7}$ factors were recently considered by Kimura in the F-theory context [91]. For example, the first case considered there has $\alpha_{4}=\left(t-x_{1}\right)^{3}\left(t-x_{2}\right)^{3}\left(t-x_{3}\right)^{2} \prod_{i=1}^{8}\left(u-y_{i}\right)$, where $t, u$ are local coordinates on the base $\mathbb{F}_{0}=\mathbb{P}^{1} \times \mathbb{P}^{1}$, giving a gauge group $\left(E_{7}^{2} \times \mathrm{SO}(7) \times \mathrm{SU}(2)^{8}\right) / \mathbb{Z}_{2}$. The other models come from combining loci of the $\mathrm{SU}(2)$ factors to form further $\mathrm{SO}(7)$ or $E_{7}$ factors in 
the group. While these models have $(4,6)$ points corresponding to SCFTs coupled to the $\mathrm{SO}(7)$ and $E_{7}$ factors, the analysis of Kimura suggests that interpreting these loci as bi-charged matter may nonetheless give solutions to the anomaly cancellation conditions. The notion that $(\mathbf{5 6}, \mathbf{2})$ matter transforming under $E_{7} \times \mathrm{SU}(2)$ may be realized in F-theory at apparent superconformal loci was also considered previously in [92]. As pointed out there, the existence of a heterotic dual with such matter suggests that there is a sensible interpretation of these constructions in the F-theory context. It would be interesting to investigate this kind of exotic matter structure further.

\section{$4.2 \mathbb{Z}_{3}$ torsion: $\mathrm{SU}(3), E_{6}, \mathrm{SU}(6)$ (but not $\mathrm{SU}(9)$ )}

A Weierstrass model with $\mathbb{Z}_{3}$ torsion takes the form $[27,83]$

$$
\begin{aligned}
f & =-\alpha_{1}^{4} / 48+\alpha_{1} \alpha_{3} / 2 \\
g & =\alpha_{1}^{6} / 864-\alpha_{1}^{3} \alpha_{3} / 24+\alpha_{3}^{2} / 4 \\
\Delta & =\alpha_{3}^{3} \tilde{\Delta} .
\end{aligned}
$$

This implies at least an $\mathfrak{s u ( 3 )}$ algebra. This form of the Weierstrass model can be understood geometrically by noting that an elliptic curve only has a point $p$ satisfying $3 p=p+p+p=0$ when the point $p$ is an inflection point that hits the tangent at that point three times, in which case the Weierstrass model can be written in the form $y^{2}+a_{1} y x+a_{3} y=x^{3}$.

As for $\mathrm{SU}(2)$, we can easily prove that a model with algebra $\mathfrak{s u}(3)$ takes the form (4.19) when there is no fundamental matter and $-a \cdot b>0$, as predicted by the massless charge sufficiency conjecture. The general form of a Weierstrass model with $\mathfrak{s u}(3)$ algebra takes the form [68]

$$
\begin{aligned}
f & =-\frac{1}{48} \phi_{0}^{4}+\frac{1}{2} \phi_{0} \psi_{1} \sigma+f_{2} \sigma^{2} \\
g & =\frac{1}{864} \phi_{0}^{6}-\frac{1}{24} \phi_{0}^{3} \psi_{1} \sigma+\left(\frac{1}{4} \psi_{1}^{2}-\frac{1}{12} \phi_{0}^{2} f_{2}\right) \sigma^{2}+g_{3} \sigma^{3} .
\end{aligned}
$$

The absence of fundamental matter dictates that $(-3 a-b) \cdot b=0$. The classes of $f_{2}, g_{3}$ are $\left[f_{2}\right]=-4 a-2 b,\left[g_{3}\right]=-6 a-3 b$, so these are both ineffective and $f_{2}=g_{3}=0$. This gives exactly the form of (4.19) with $\alpha_{1}=\phi_{0}, \alpha_{3}=\psi_{1} \sigma$, confirming that all models with no fundamental matter and $-a \cdot b>0$ have $\mathbb{Z}_{3}$ torsion, so that the massless charge sufficiency conjecture holds for $\mathfrak{s u}(3)$.

The simplest class of $\mathfrak{s u}(3)$ models of this form are those where the divisor $\sigma$ supporting the gauge factor has the maximum class $-3 K_{B}$. In this case $\alpha_{3}=c \sigma$ with $c$ a constant. In the case where $\sigma$ is irreducible, this gives a $6 \mathrm{D}$ F-theory model with gauge group $\mathrm{SU}(3) / \mathbb{Z}_{3}$, and only adjoint matter fields (with multiplicity $g=1+3 K_{B}^{2}$ ). For $T=0$, this reproduces the only anomaly-free theory with $\mathfrak{s u}(3)$ gauge algebra and only adjoint matter.

As in the $\mathbb{Z}_{2}$ case, when we tune an $\mathrm{SU}(3)$ factor on a large enough divisor $\sigma$ that there are no terms in $f, g$ of higher order in $\sigma$, then we get the Weierstrass form (4.19) with $\alpha_{3}=\tau \sigma$ and there is another gauge factor of $\mathrm{SU}(3)$ on $\tau$ (multiple factors if $\tau$ is 
reducible), for a global gauge group $(\mathrm{SU}(3) \times \mathrm{SU}(3)) / \mathbb{Z}_{3}$. As we found for the analogous $\mathfrak{s u}(2)$ models, this construction also points to further classes of anomaly-free models that have no known F-theory construction, such as for example the $T=0$ model with gauge group $\mathrm{SU}(3)$, anomaly coefficient $b=8$, and 24 fields in the fundamental of SU(3) (along with 21 adjoint fields), since e.g. attempting to tune such a Weierstrass model automatically gives a second $\mathrm{SU}(3)$ factor, only bifundamental and adjoint fields, and a global gauge group $(\mathrm{SU}(3) \times \mathrm{SU}(3)) \mathbb{Z}_{3}$.

One can again in this case construct various product models by taking $\alpha_{3}$ to be reducible when $\left[\alpha_{3}\right]=-3 K_{B}$.

We can also consider models with the group $E_{6}$, which has a center $\mathbb{Z}_{3}$. Like in the $E_{7}$ case above, we have

$$
\begin{aligned}
f & =\sigma^{3} \tilde{f} \\
g & =\sigma^{4} \tilde{g} \\
\Delta & =27 \sigma^{8} \tilde{g}^{2} .
\end{aligned}
$$

Fundamental matter appears at the locus where $\tilde{g}=0$. We can prove the massless charge sufficiency conjecture for $E_{6}$ in a similar fashion to the preceding cases. The absence of fundamental matter is equivalent to the condition $(-3 a-2 b) \cdot b=0$. It follows that $\tilde{f}=0$, and there can be no higher-order terms in $\tilde{g}$ in an expansion in $\sigma$. For Weierstrass models of the form (4.21), with no terms in $g$ of order $\sigma^{5}$ the monodromy condition for $E_{6}$ is that $\tilde{g}$ is a perfect square $\tilde{g}=\chi^{2}$ (otherwise we have a group $F_{4}$ ). When this condition is satisfied, (4.21) takes the form (4.19) with $\alpha_{3}=\sigma^{2} \chi$. Thus, whenever we have a group $E_{6}$ with no fundamental matter and $-a \cdot b>0$ it follows that the Weierstrass model has the $\mathbb{Z}_{3}$ torsion form (4.19). This proves the massless charge sufficiency conjecture for the group $E_{6}$.

In the simplest example of $E_{6}$ theories with $\mathbb{Z}_{3}$ torsion, $\tilde{g}$ is a constant, which occurs when $\sigma$ is a section of $-3 K_{B} / 2$. This is possible, for example, on the base $\mathbb{F}_{0}=\mathbb{P}^{1} \times \mathbb{P}^{1}$, when $\sigma$ is a curve of bidegree $(3,3)$. In this case $\tilde{f}=0$, and we match the Weierstrass form (4.19) with $\alpha_{1}=0, \alpha_{3}=\sqrt{4 \tilde{g}} \sigma^{2}$, precisely corresponding to the case where the $E_{6}$ theory has only adjoint matter.

Finally, we consider one case with exotic matter: $\mathrm{SU}(6)$ with only matter in the adjoint and 3-index antisymmetric representation. These matter representations preserve the $\mathbb{Z}_{3}$ subgroup of the discrete center $\mathbb{Z}_{6}$, so that the global form of the gauge group is $\mathrm{SU}(6) / \mathbb{Z}_{3}$. From anomaly cancellation, this is possible when $-9 a \cdot b=6 b \cdot b$. The general form of Weierstrass model with $\mathrm{SU}(6)$ gauge group and 3-index antisymmetric representations was worked out in [68] As shown in [93], for such models with no antisymmetric matter, a Tate-type tuning can be realized through the general Weierstrass form

$$
y^{2}+a_{1} x y+\tilde{a}_{3} \sigma^{2} y=x^{3},
$$

where fundamental matter occurs at the locus $\tilde{a}_{3}=\sigma=0$, so that the absence of fundamental matter occurs precisely when $(-3 a-2 b) \cdot b=0$. This Tate tuning matches with the $\mathbb{Z}_{3}$ Weierstrass form (4.19). Here again (omitting the details, which are similar to the 
other cases) the massless charge sufficiency hypothesis holds and we have the appropriate Mordell-Weil torsion group. In the simplest cases, this corresponds to a situation in F-theory where $[\sigma]=-3 K_{B} / 2$, which can be realized for example on $\mathbb{F}_{0}$.

A similar construction would seem to possible for an $\mathrm{SU}(9)$ theory, where we use $a_{3}=\tilde{a}_{3} \sigma^{3}$, with $[\sigma]=-K_{B}$. This would seem to correspond to the structure needed for an $\mathrm{SU}(9)$ theory with $\mathrm{SU}(9) / \mathbb{Z}_{3}$ symmetry. This could be imagined for example to occur in a model in which all the matter is in 3-index antisymmetric representations. As discussed in e.g. [90], however, this representation only seems to arise in the context of (4, 6) points associated with an SCFT. This can be seen in the Weierstrass model from the vanishing of $f, g, \Delta$ to orders $4,6,12$ at the locus $a_{1}=\sigma=0$. Nonetheless, this suggests that these SCFT loci may have a natural interpretation as exotic SU(9) matter in the Ftheory context that preserves the $\mathbb{Z}_{3}$ discrete central symmetry, like the $E_{7} \times \mathrm{SU}(2)$ matter discussed above that preserves a $\mathbb{Z}_{2}$ symmetry. How the anomaly equations work out in this case, however, is rather unclear, ${ }^{15}$ and a further analysis of this class of models is left for further investigation.

\subsection{Higher torsion groups}

\subsection{1 $\mathbb{Z}_{4}$}

Continuing in this vein we next consider the torsion group $\mathbb{Z}_{4}$. In this case we cannot simply realize this through a theory with gauge group $\mathrm{SU}(4) / \mathbb{Z}_{4}$ and only adjoint matter. We can see this since from the anomaly conditions the number of two-index antisymmetric fields on a curve $C$ associated with anomaly coefficient $b$ is $-a \cdot b$, while the number of fundamental fields is $-8 a \cdot b-4 b \cdot b$; these can only both vanish if $b \cdot b=-a \cdot b=0$, which is an exceptional case where the condition $-a \cdot b>0$ does not hold. The same kind of argument holds for all higher $\mathrm{SU}(N)$, so we do not expect any F-theory models with a gauge group of only $\mathrm{SU}(N) / \mathbb{Z}_{N}$ for $N>3$, except when $-a \cdot b=0$, so the massless charge sufficiency condition holds trivially in this class of cases.

Similarly, we do not expect any models with torsion $\mathbb{Z}_{4}$ from a gauge algebra $\mathfrak{s o}(4 n+2)$. The $\mathbb{Z}_{4}$ center is broken by fundamental fields, with multiplicity $(-(2 n-1) a-(2 n-2) b) \cdot b$ and also by spinor fields, with multiplicity $(-2 a-b) \cdot b$. These multiplicities can only both vanish when $-a \cdot b=b \cdot b$, which is again one of the exceptional cases, so we again can have no counterexamples to the massless charge sufficiency conjecture.

\footnotetext{
${ }^{15}$ In particular, if we take the base $\mathbb{P}^{2}$ and $\sigma$ to be a cubic, the generic matter content from anomaly cancellation would be -9 fundamental fields and +9 two-index antisymmetric fields. These cannot be exchanged for only 3-index antisymmetric matter, however, as the 3-index antisymmetric representation is anomaly equivalent to 52 -index antisymmetric fields and -14 fundamental fields. Thanks to Patrick Jefferson for discussions on this issue.
} 
We can identify some models with torsion $\mathbb{Z}_{4}$ by analyzing the Weierstrass model form with this torsion $[27]$

$$
y^{2}+a_{1} x y+a_{1} a_{2} y=x^{3}+a_{2} x^{2},
$$

which gives

$$
\begin{aligned}
f & =-\frac{1}{48} a_{1}^{4}+\frac{1}{3} a_{1}^{2} a_{2}-\frac{1}{3} a_{2}^{2} \\
g & =\frac{1}{864}\left(a_{1}^{2}-8 a_{2}\right)\left(a_{1}^{4}-16 a_{1}^{2} a_{2}-8 a_{2}^{2}\right) \\
\Delta & =-\frac{1}{16} a_{1}^{2} a_{2}^{4}\left(a_{1}^{2}-16 a_{2}\right) .
\end{aligned}
$$

From this we see that this torsion arises when the gauge algebra contains at least $\mathfrak{s u}(2) \oplus$ $\mathfrak{s u}(4)$. In particular, when $a_{1}, a_{2}$ are irreducible divisors in the classes $-K_{B},-2 K_{B}$ we get a gauge group $(\mathrm{SU}(2) \times \mathrm{SU}(4)) / \mathbb{Z}_{4}$. Consideration of anomalies shows that the spectrum of an $\mathrm{SU}(2)$ gauge group on a divisor of class $-K_{B}$ has $6 K_{B} \cdot K_{B}$ fields in the fundamental representation, and the spectrum of an $\mathrm{SU}(4)$ gauge group on a divisor of class $-2 K_{B}$ has $2 K_{B} \cdot K_{B}$ fields in the two-index antisymmetric representation. The curves $a_{1}, a_{2}$ intersect at $2 K_{B} \cdot K_{B}$ points, so each of these points must support a half-hypermultiplet in the $(\mathbf{2}, \mathbf{6})$ representation of $\mathfrak{s u}(2) \oplus \mathfrak{s u}(4)$, which is invariant under $\mathbb{Z}_{4}$. Indeed, while we have not done an exhaustive analysis, it does not seem possible to construct any other anomaly-consistent theory that has a spectrum invariant under a $\mathbb{Z}_{4}$ subgroup, other than by making the $\mathrm{SU}(2)$ and SU(4) loci $a_{1}$ and/or $a_{2}$ reducible. Note in particular that trying to factor $a_{2}=\alpha^{2}$ to get an $\mathrm{SU}(8)$ factor gives $(4,6)$ points at the locus $a_{1}=\alpha=0$ unless $-a \cdot b=0$, which is an exceptional case. Similarly, factoring $a_{2}=a_{1} \beta$, which naively would give a theory with gauge group $\mathrm{SU}(4) \times \mathrm{SO}(12)$ gives $(4,6)$ points at the locus $a_{1}=\beta=0$ unless we are in an exceptional case.

\subsection{2 $\mathbb{Z}_{2} \oplus \mathbb{Z}_{2}$}

In this case the Weierstrass model comes from a Tate form

$$
y^{2}=x\left(x-b_{2}\right)\left(x-c_{2}\right)
$$

giving

$$
\begin{aligned}
f & =\frac{1}{3}\left(b_{2} c_{2}-b_{2}^{2}-c_{2}^{2}\right) \\
g & =-\frac{1}{27}\left(b_{2}+c_{2}\right)\left(b_{2}-2 c_{2}\right)\left(2 b_{2}-c_{2}\right) \\
\Delta & =-b_{2}^{2} c_{2}^{2}\left(b_{2}-c_{2}\right)^{2}
\end{aligned}
$$

The only single-factor groups that have a center $\mathbb{Z}_{2} \oplus \mathbb{Z}_{2}$ are the groups $\operatorname{spin}(4 n)$, with algebra $\mathfrak{s o}(4 n)$. To keep this center unbroken we must have no spinor matter, of multiplicity $(-2 a-b) \cdot b$, and no fundamental matter, of multiplicity $(-(2 n-2) a-(2 n-3) b) \cdot b$. This is only possible when $n=2$, i.e. for $\mathfrak{s o}(8)$. From the point of view of Weierstrass 
models, the absence of spinor matter, as discussed in section 4.1.5, is only possible with $-a \cdot b>0$ when we have an $\mathfrak{s o}(8)$ algebra and a Weierstrass form (4.18) with $f_{3}=g_{4}=0$, and $f_{2}=c A^{2}, g_{3}=c^{\prime} A^{3}$. This corresponds to a model with $\mathrm{SO}(8)$ gauge group and no fundamental or spinor matter. This has the form (4.26), where $c_{2}, b_{2}$ are proportional to $A$, and thus has the necessary $\mathbb{Z}_{2} \oplus \mathbb{Z}_{2}$ torsion, so the massless matter sufficiency conjecture is satisfied for all single-factor groups with torsion $\mathbb{Z}_{2} \oplus \mathbb{Z}_{2}$.

More generally, (4.26) gives a gauge group of $\mathrm{SU}(2)^{3} /\left(\mathbb{Z}_{2} \oplus \mathbb{Z}_{2}\right)$, where the three $\mathrm{SU}(2)$ factors are tuned on divisors $b_{2}, c_{2}, b_{2}-c_{2}$ in the class $-2 K_{B}$. The anomaly-free spectrum consists of $4 K_{B} \cdot K_{B}$ half-hypermultiplet bifundamental fields in each pair of SU(2) factors. The $\mathbb{Z}_{2}$ actions act on pairs of SU(2) factors; note that the product of e.g. the $\mathbb{Z}_{2}$ actions on factors $1+2$ and $2+3$ combine to give the $\mathbb{Z}_{2}$ action on the $1+3$ factors.

As in the other cases, we can get more complicated models by taking $b_{2}, b_{3}$ in factored form. For example, setting $b_{2}=\beta_{1}^{2}$ gives a theory with $(\mathrm{SU}(4) \times \mathrm{SU}(2) \times \mathrm{SU}(2)) /\left(\mathbb{Z}_{2} \oplus \mathbb{Z}_{2}\right)$ symmetry.

\subsubsection{Other discrete torsion groups}

For the groups $\mathbb{Z}_{5}, \mathbb{Z}_{6}, \mathbb{Z}_{4} \oplus \mathbb{Z}_{2}$, and $\mathbb{Z}_{3} \oplus \mathbb{Z}_{3}$ there are no natural realizations of these torsion groups from anomaly-free models with single-factor gauge groups. The only place these might be realized from a single group factor is through $\mathrm{SU}(5) / \mathbb{Z}_{5}$ or $\mathrm{SU}(6) / \mathbb{Z}_{6}$, but there are no anomaly-free models with these gauge groups and no fundamental or two-index antisymmetric matter where $-a \cdot b>0$.

Indeed, it turns out that there are no good Weierstrass models with these Mordell-Weil torsion forms other than models with $(4,6)$ points and models where gauge group factors are tuned on divisors that violate the massless charge sufficiency condition $-a \cdot b>0$, which we discuss further in section 6 .

As an example, for $\mathbb{Z}_{5}$, the Weierstrass model takes the form [27]

$$
\begin{aligned}
f & =\frac{1}{6} a_{1} b_{1}^{3}-\frac{1}{48} a_{1}^{4}+\frac{1}{3} a_{1}^{2} b_{1}^{2}-\frac{1}{3} b_{1}^{4}-\frac{1}{6} a_{1}^{3} b_{1} \\
g & =\frac{1}{864}\left(a_{1}^{2}-2 a_{1} b_{1}+2 b_{1}^{2}\right)\left(a_{1}^{4}+14 a_{1}^{3} b_{1}+26 a_{1}^{2} b_{1}^{2}-116 a_{1} b_{1}^{3}+76 b_{1}^{4}\right) \\
\Delta & =\frac{1}{16}\left(a_{1}^{2}+9 a_{1} b_{1}-11 b_{1}^{2}\right)\left(a_{1}-b_{1}\right)^{5} b_{1}^{5} .
\end{aligned}
$$

Here the parameters $a_{1}, b_{1}$ are both sections of the line bundle $\mathcal{O}\left(-K_{B}\right)$. When $K_{B} \cdot K_{B} \neq 0$, then there are points where both $a_{1}$ and $b_{1}$ vanish. At such points, $f, g, \Delta$ vanish to orders $4,6,12$, corresponding to the appearance of an SCFT in the $6 \mathrm{D}$ theory so that there is no simple interpretation in terms of gauge groups and local matter fields. We do not consider such constructions here, although again the question of interpreting SCFT loci as exotic matter fields begs further investigation. Thus, the only theories we consider that admit discrete $\mathbb{Z}_{5}$ Mordell-Weil torsion are those where gauge group factors SU(5) or larger are tuned on divisors satisfying $-a \cdot b=b \cdot b=0$. The same is true for the other discrete torsion cases mentioned above: $\mathbb{Z}_{6}, \mathbb{Z}_{4} \oplus \mathbb{Z}_{2}, \mathbb{Z}_{3} \oplus \mathbb{Z}_{3}$. 


\section{$5 \quad \mathrm{U}(1)$ gauge factors}

All the cases considered in the previous section involved nonabelian gauge factors, associated with local Kodaira-type fiber singularities arising at codimension one loci in the base. We have given a fairly comprehensive treatment of such factors. Abelian U(1) factors, however, associated with the infinite $\mathbb{Z}^{r}$ part of the Mordell-Weil group, are much less well understood. The assertion of massless charge sufficiency for the continuous part of the gauge group states that the massless charges in the theory must span the full charge lattice, even when such $U(1)$ factors are included. We conjecture that this holds for any $\mathrm{U}(1)$ factor with anomaly coefficient $\tilde{b}$ satisfying $-a \cdot \tilde{b}>0$. Because the matter structure of $\mathrm{U}(1)$ theories is not as well understood, it is harder to get a complete picture of this class of theories. Here we consider only a single case: a situation where there appears to be a $U(1)$ factor with massless fields only of charge 2 . We show that in fact this case satisfies the massless charge sufficiency hypothesis through the appearance of an additional section that is the correct generator for the Mordell-Weil group. A brief discussion of further cases involving $\mathrm{U}(1)$ gauge factors is given in section 5.2

\section{$5.1 \mathrm{U}(1)$ and "charge 2" matter}

We thus now consider the case of the Morrison-Park model that appears to have only charge 2 matter under the $\mathrm{U}(1)$ field (i.e., no matter of charge 1). We first recall the general form of the Morrison-Park [94] model, which gives a generic F-theory compactification with a $\mathrm{U}(1)$ gauge field and charges $q=1,2$, using the notation and conventions of [95] (but using $\hat{b}$ in place of $b$ to avoid confusion with the anomaly coefficient $b$ for nonabelian groups)

$$
y^{2}=x^{3}+\left(-\frac{1}{3} e_{2}^{2}+e_{1} e_{3}-e_{0} \hat{b}^{2}\right) x+\left(\frac{1}{3} e_{1} e_{2} e_{3}+\frac{2}{3} e_{0} e_{2} \hat{b}^{2}-\frac{1}{4} e_{1}^{2} \hat{b}^{2}-e_{0} e_{3}^{2}-\frac{2}{27} e_{2}^{3}\right) .
$$

The parameters $e_{i}$ are sections of particular line bundles parameterized by the single class $L$ :

$$
\begin{aligned}
{\left[e_{0}\right] } & =2 L \\
{\left[e_{1}\right] } & =-K_{B}+L \\
{\left[e_{2}\right] } & =-2 K_{B} \\
{\left[e_{3}\right] } & =-3 K_{B}-L \\
{[\hat{b}] } & =-2 K_{B}-L .
\end{aligned}
$$

The anomaly coefficient $\tilde{b}$ of the resulting $\mathrm{U}(1)$ model is associated with the class $2\left[e_{3}\right]$. We are interested in particular in two special cases: in the first special case, only massless matter with charge $q=1$ appears. This occurs when $L=-2 K_{B}$, so that $\hat{b}$ is a constant, which we can rescale so that $\hat{b}=1$. In the second special case, where for clarity we denote the parameters by $\check{e}_{i}$, only massless matter with charge $q=2$ appears. This occurs when $L=K_{B}$, in which case $\check{e}_{0}=0$ must vanish identically as $2 L=2 K_{B}$ is not effective, and $\check{e}_{1}$ is a constant, which we can take to be $\check{e}_{1}=1$. In these two cases we have $\tilde{b}=-2 a, \tilde{b}=-4 a$. As long as $T<9$, these give models satisfying $-a \cdot \tilde{b}>0, \tilde{b} \cdot \tilde{b}>0$. 
Our assertion is that the Morrison-Park models in these two special cases are precisely equivalent. That is, the $q=2$ model with

$$
\begin{aligned}
& f=\check{e}_{3}-\check{e}_{2}^{2} / 3 \\
& g=-\left(-\frac{2}{27} \check{e}_{2}^{3}+\frac{1}{3} \check{e}_{2} \check{e}_{3}-\frac{1}{4} \check{b}^{2}\right)
\end{aligned}
$$

is equivalent to the $q=1$ model with

$$
\begin{aligned}
f & =-\frac{1}{3} e_{2}^{2}+e_{1} e_{3}-e_{0} \\
g & =-\left(\frac{1}{3} e_{1} e_{2} e_{3}+\frac{2}{3} e_{0} e_{2}-\frac{1}{4} e_{1}^{2}-e_{0} e_{3}^{2}-\frac{2}{27} e_{2}^{3}\right) .
\end{aligned}
$$

This equivalence occurs because the natural section for the $q=2$ special case of MorrisonPark is actually not the generating section of the Mordell-Weil group; as noted in the final appendix of [94], this model contains an additional section corresponding to the generator of the $\mathrm{U}(1)$, which matches the natural section in the $q=1$ special case. ${ }^{16}$ To show this equivalence explicitly, we identify the relationship between the parameters in the two models to be

$$
\begin{aligned}
\check{b} & =-e_{1}+2 e_{2} e_{3}-2 e_{3}^{3} \\
\check{e}_{2} & =-2 e_{2}+3 e_{3}^{2} \\
\check{e} & =-e_{0}+e_{2}^{2}+e_{1} e_{3}-4 e_{2} e_{3}^{2}+3 e_{3}^{4} .
\end{aligned}
$$

Explicit use of these relations shows through a short calculation that the models are indeed equivalent as stated. Furthermore, by taking $e_{3}$ to be an arbitrary section of $\mathcal{O}\left(-K_{B}\right)$, we can solve the equations for $e_{2}, e_{1}, e_{0}$ successively, so that for every $q=2$ type model there is a family of $q=1$ type models associated with the allowed values of $e_{3}$, representing an over-parameterization of the associated Weierstrass models.

This shows that the models that appear to have only $q=2$ charges under the $\mathrm{U}(1)$ gauge symmetry are actually equivalent to models with the fundamental charge $q=1$. Thus, the " $q=2$ " type models actually have an additional section $\sigma$, as expected through Poincaré duality and the massless charge sufficiency hypothesis; this section satisfies $\sigma \cdot C=$ 1 for the curves that appear to have charge 2 in the usual Morrison-Park formulation.

While this explicit demonstration of equivalence is mathematically efficient, it does not explain much about the geometry. We make some additional observations that help to clarify this story.

First, we note that the $q=1$ case, where $\hat{b}$ is a constant that can be taken to be unity, is the only case where we can shift the section identified by Morrison-Park

$$
\left[x_{1}, y_{1}, z_{1}\right]=\left[e_{3}^{2}-\frac{2}{3} \hat{b}^{2} e_{2},-e_{3}^{3}+\hat{b}^{2} e_{2} e_{3}-\frac{1}{2} \hat{b}^{4} e_{1}, \hat{b}\right]
$$

\footnotetext{
${ }^{16}$ We would also like to thank Remke Kloosterman and Nikhil Raghuram for discussions regarding this additional section.
} 
to the origin without involving rational functions with a nontrivial denominator. Performing the shift

$$
x \rightarrow x+x_{1}, \quad y \rightarrow y+y_{1}
$$

the $q=1$ Weierstrass model takes the form

$$
y^{2}+a_{3} y=x^{3}+a_{2} x^{2}+a_{4} x
$$

where the section is now located at $x=y=0$, and we identify (setting $\hat{b}=1$ )

$$
\begin{aligned}
& a_{2}=3 x_{1} \\
& a_{3}=2 y_{1} \\
& a_{4}=-e_{0}+e_{2}^{2}+e_{1} e_{3}-4 e_{2} e_{3}^{2}+3 e_{3}^{4} .
\end{aligned}
$$

If we now complete the square and cube in the Weierstrass model (5.12), we find that the resulting Weierstrass normal form has precisely the form of (5.7), with the identifications

$$
\begin{aligned}
\check{b} & =a_{3} \\
\check{e}_{2} & =a_{2} \\
\check{e}_{3} & =a_{4} .
\end{aligned}
$$

On the other hand, completing the square in this way must return us to the original $q=1$ Weierstrass model. Combining (5.14) and (5.13), we reproduce the relations of (5.9), giving a simple way of understanding the formulae appearing in this relationship.

To confirm this picture, we can check explicitly that the natural section of the $q=2$ Morrison-Park model corresponds under the elliptic curve addition law to twice the natural section of the $q=1$ model. In terms of the parameterization (5.12), where the section $s=\left[x_{1}, y_{1}, 1\right]$ has been shifted to the origin we can compute $s+s$ using the elliptic curve law. The tangent to (5.12) at the point $s$ is given by the line $y=a_{4} x / a_{3}$. This line intersects the cubic at the third point $\tilde{r}=\left(\tilde{x}, a_{4} \tilde{x} / a_{3}\right)$, where $\tilde{x}=-a_{2}+a_{4}^{2} / a_{3}^{2}$. This represents the point $-2 s$ on the elliptic curve. To find $2 s$, we identify the other point on the cubic that intersects a vertical line passing through this point, which is

$$
2 s \sim\left(-a_{2}+a_{4}^{2} / a_{3}^{2},-a_{3}+a_{2} a_{4} / a_{3}-a_{4}^{3} / a_{3}^{3}\right) .
$$

Shifting $x, y$ to go back to the original Weierstrass normal form, and scaling by appropriate powers of $a_{3}$, we see that this precisely reproduces the expected Morrison-Park section

$$
2 s \rightarrow\left[-\frac{2}{3} a_{2} a_{3}^{2}+a_{4}^{2},-a_{3}^{4} / 2+a_{2} a_{4} a_{3}^{2}-a_{4}^{3}, a_{3}\right] .
$$

So we find that indeed the natural section of the " $q=2$ " Morrison-Park model is twice the generating section identified from the " $q=1$ " form of the same model, confirming the massless charge sufficiency hypothesis in this situation. 


\subsection{Other aspects of $U(1)$ factors}

We have considered here only a single example of a theory with a U(1) factor that appears naively to violate the massless charge sufficiency conjecture. There are many other similar models that satisfy anomaly cancellation conditions [14]; for example, any model with 54 matter fields with each of the $\mathrm{U}(1)$ charges $a q, a p, a(q+p)$ satisfies anomaly cancellation, for arbitrary integers $a>1, p, q$, but violates the massless charge sufficiency conjecture. Few such models have known F-theory constructions, however, and F-theory in principle can only realize a finite subset of this infinite class of models. In particular, explicit Weierstrass models are only known for $\mathrm{U}(1)$ charges up to $q=3,4$ [96, 97], though general arguments suggest that charges as large as $q=6[98,99]$ or even $q=21$ [100] should be possible in 6D F-theory models. It was shown in [19] that the above-mentioned infinite family of $\mathrm{U}(1)$ models would appear to violate the automatic enhancement conjecture, and indeed lead to a larger gauge group in the simplest cases where F-theory realizations are possible, but there are other more complicated infinite families where this story is even less clear. It would be interesting to explore further if and how massless charge sufficiency is realized for models that apparently have matter content where all charges under a given $\mathrm{U}(1)$ factor are multiples of an integer $a>2$.

Another interesting class of models that we have not addressed in the preceding sections involve situations where the gauge group has nonabelian and abelian factors, and may have a discrete quotient, such as $\mathrm{U}(N)=(\mathrm{SU}(N) \times \mathrm{U}(1)) / \mathbb{Z}_{N}$. Some analysis of the charges in such situations were considered in $[58,59,61]$. Models of this type where the gauge group contains $\mathrm{SU}(3) \times \mathrm{SU}(2) \times \mathrm{U}(1)$ factors, with the possibility of a discrete quotient by $\mathbb{Z}_{6}$, have recently been considered in $[56,57,60,62-65]$. In this situation in particular, for example, however, as discussed in more detail in the appendix, the fundamental group of the gauge group $(\mathrm{SU}(3) \times \mathrm{SU}(2) \times \mathrm{U}(1)) / \mathbb{Z}_{6}$ is just $\mathbb{Z}$, and there is no Mordell-Weil torsion. The generic matter representations under this group, which include the charges of the MSSM [81], generate the full charge lattice of the quotient group, and the massless charge sufficiency hypothesis is satisfied for the models that have been considered of this type.

\section{Exceptions to massless charge sufficiency}

As discussed in section 3 , there are two distinct classes of exceptions to the condition $-a \cdot b>0$ that we conjecture leads to massless charge sufficiency. The first class corresponds to nonabelian factors where $-a \cdot b<0$, and the second class corresponds to abelian or nonabelian factors where $-a \cdot b=b \cdot b=0$. We consider these two cases of exceptions in turn.

\subsection{Non-Higgsable nonabelian factors: exceptional cases with $-a \cdot b<0$}

The exceptional cases with $-a \cdot b<0$ correspond to F-theory models where the base $B$ contains rational curves with $-K_{B} \cdot C<0$. Multiples of the anticanonical class $-n K_{B}$ must contain one or more multiples of the rigid curve $C$, which has self-intersection $C \cdot C=$ $-K_{B} \cdot C-2$, as a component. It follows that the Weierstrass coefficients $f, g$ must vanish 
over $C$ to sufficiently high degrees to force at least a type IV vanishing associated with an $\mathfrak{s u}(3)$ algebra. The configurations of curves of this type that are allowed in F-theory models without $(4,6)$ loci are known as "non-Higgsable clusters", since the associated gauge groups cannot be broken by giving a Higgs VEV to any of the matter fields without breaking supersymmetry, and were classified in [75].

Many of the non-Higgsable clusters give single gauge factors with no matter. In particular, curves of self-intersection $-3,-4,-6,-8,-12$ give rise to the gauge algebras $\mathfrak{s u}(3), \mathfrak{s o}(8), \mathfrak{e}_{6}, \mathfrak{e}_{7}$, and $\mathfrak{e}_{8}$. While there is no matter charged under any of these gauge fields, in each of these cases the constraints on the Weierstrass model are completely local and in general there is no Mordell-Weil torsion. As a simple example of this consider the SU(3) non-Higgsable gauge factor that arises on the -3 curve $S$ in the Hirzebruch surface $\mathbb{F}_{3}$. Standard toric methodology (see, e.g., [101]) gives an explicit description of $f, g$ in terms of monomials associated with lattice points in the 4th and 6th polar polytopes to the polytope associated with the toric base $\mathbb{F}_{3}$. Taking $\sigma$ to be a coordinate that vanishes on $S$, we have

$$
\begin{aligned}
& f=f_{2,2} \sigma^{2}+f_{3,5} \sigma^{3}+\cdots \\
& g=g_{2,0} \sigma^{2}+g_{3,3} \sigma^{3}+\cdots
\end{aligned}
$$

where the second index in the subscript denotes the degree of the associated coefficient considered (locally) as a function of the coordinate on the rational curve $S$. The coefficients of each of the monomials in this expansion can be chosen independently, as $f, g$ are general sections of $-4 K_{B},-6 K_{B}$ respectively. It is easy to compute that the numbers of independent degrees of freedom in $f, g$ are 84,176 respectively (this is closely related to the total number of uncharged hypermultiplets in the theory, 252). On the other hand, for a Weierstrass model with $\mathbb{Z}_{3}$ torsion, which must have the form (4.19), the parameters $\alpha_{1}, \alpha_{3}$ are sections of $-K_{B},-3 K_{B}$ and have only 9,51 degrees of freedom respectively. Thus, the generic Weierstrass model over $\mathbb{F}_{3}$ has an $\mathfrak{s u}(3)$ gauge algebra over $S$ but does not have Mordell-Weil torsion, so the gauge group is indeed SU(3). Since there is no matter this represents a case in which the massless charge sufficiency condition does not hold. Similarly, since the other single non-Higgsable gauge group factors without matter result from purely local effects in the geometry they do not generically correspond to Weierstrass models with Mordell-Weil torsion, which is a global effect. The other single non-Higgsable gauge factors are therefore $\operatorname{spin}(8), E_{6}, E_{7}$, and $E_{8}$. All of these except for the $E_{8}$ case represent exceptions to the massless charge sufficiency condition.

Over a -5 curve there is a gauge factor $F_{4}$. This does not have a nontrivial discrete center so there is no possibility of Mordell-Weil torsion. Over a -7 curve there is a $E_{7}$ gauge group with a single multiplet of matter in the fundamental (56). Because the matter breaks the central $\mathbb{Z}_{2}$, there is no possibility for Mordell-Weil torsion.

There are also three non-Higgsable clusters containing a combination of -3 and -2 curves. A cluster containing one each of these curves, intersecting at a point, supports a gauge group $G_{2} \times \mathrm{SU}(2)$, with a half-hypermultiplet of matter in the $(7,2)$ representation. This breaks the $\mathbb{Z}_{2}$ central symmetry of the $\mathrm{SU}(2)$ factor so there is no possibility of torsion. The same situation holds for a chain of three curves of self-intersections $-3,-2,-2$. In 
the final case, however, we have a chain of three curves of self-intersections $-2,-3,-2$, supporting a gauge group $\mathrm{SU}(2) \times \mathrm{SO}(7) \times \mathrm{SU}(2)$. There is a matter field jointly charged under the $\mathrm{SO}(7)$ factor and each of the $\mathrm{SU}(2)$ factors, in the $(8,2)$ representation. This matter breaks two of the three combinations of $\mathbb{Z}_{2}$ factors in the center. There is, however, a third $\mathbb{Z}_{2}$ factor in the center, given by the product of all three $\mathbb{Z}_{2}$ factors, which acts trivially on all the matter in this cluster. Nonetheless, like the single gauge factors this is a local effect and there is no Mordell-Weil torsion in general, so this cluster represents another exception to massless charge sufficiency.

Finally, we point out that the $\operatorname{Spin}(8)$ on a -4 curve can be enhanced to a larger $\operatorname{Spin}(N)$ group that has fundamental matter but no spinor matter. In this case there can still be a discrete subgroup of the gauge factor that acts trivially on all the matter, but the gauge group is still $\operatorname{Spin}(N)$; this gives further exceptions to massless charge sufficiency where $-a \cdot b<0$.

This essentially exhausts all the possible exceptions in the first class.

\subsection{Geometry of exceptional cases with $-a \cdot b=b \cdot b=0$}

In this section we describe in further detail the second class of exceptions to the massless charge sufficiency hypothesis for 6D F-theory models. These exceptions arise in cases where we have a gauge factor associated with an irreducible divisor $C$ such that

$$
K_{B} \cdot C=C \cdot C=0 .
$$

Physically, in these cases the matter content matches with what we would expect in a theory with twice as much supersymmetry, where the vector supermultiplet contains a single adjoint field. For a nonabelian gauge factor this means that the matter content in the $6 \mathrm{D} \mathcal{N}=1$ theory contains a single adjoint hypermultiplet, and for an abelian $\mathrm{U}(1)$ factor it means that there is no matter charged under the $\mathrm{U}(1)$. In both cases, there is no one-loop anomaly associated with the gauge factor, and no Green-Schwarz mechanism occurs, in accord with the vanishing of the terms $K_{B} \cdot C, C \cdot C$ that appear on the left-hand side of the anomaly equations.

Geometrically, the significance of the conditions (6.3) is that the curve $C$ is a genus one curve (i.e. a two-dimensional torus) with trivial normal bundle. Locally, therefore, the geometry is like that of a flat 7-brane in 10D flat space, so it makes sense that the matter content matches with that expected of the vector supermultiplet with enhanced supersymmetry. The existence of such a genus one curve with trivial normal bundle means that, at least to first order locally, the base $B$ is itself elliptically fibered.

The simplest base $B$ that contains such a curve $C$ is the del Pezzo surface $d P_{9}$, also known as a rational elliptic surface. This surface can be thought of as an elliptic fibration over the $1 \mathrm{D}$ base $\mathbb{P}^{1}$, where instead of the 24 vanishing points of the discriminant needed to form a $\mathrm{K} 3$ surface, the discriminant vanishes only to order 12 , and $f, g$ are sections of $\mathcal{O}\left(-2 K_{\mathbb{P}^{1}}\right)$ and $\mathcal{O}\left(-3 K_{\mathbb{P}^{1}}\right)$ respectively. Other bases can also contain a curve satisfying (6.3), e.g. by blowing up a point on the base well away from $C$ (which we note is only possible when we have a degenerate surface containing curves of self-intersection -3 or below, which support non-Higgsable nonabelian gauge factors). 


\subsection{Non-Higgsable U(1) factors}

The generic elliptic fibration over a smooth rational elliptic surface gives a Calabi-Yau threefold with Hodge numbers $(19,19)$ known as a Schoen manifold. ${ }^{17}$ More generally, Schoen constructed a class of Calabi-Yau threefolds as fiber products of rational elliptic surfaces; these rational elliptic surfaces can include singular fiber configurations, giving to a range of threefold constructions. The F-theory physics associated with such constructions was analyzed in some detail in [80]. The most important feature of the Schoen manifold is the presence of extra sections of the threefold elliptic fibration at generic points in the moduli space. The generic Schoen manifold has 9 independent sections, leading to a fibration with Mordell-Weil rank 8. Physically, these sections give rise in F-theory to $\mathrm{U}(1)$ gauge factors that are (supersymmetrically) non-Higgsable; there are no massless fields charged under these $\mathrm{U}(1)$ factors, and the $\mathrm{U}(1)$ factors are present everywhere in the smooth moduli space. These kinds of non-Higgsable U(1) factors were analyzed in [78-80]. When the fiber product includes rational elliptic surfaces with singular fibers, the sections can combine into Kodaira singularities of the threefold associated with nonabelian gauge factors; in this subsection we focus on the generic non-Higgsable U(1) factors and look at the associated nonabelian factors in the next subsection.

From the complete absence of massless fields charged under such a $\mathrm{U}(1)$ factor, it is clear that they violate the massless charge sufficiency conjecture. It is interesting that such fields can only arise in the "higher supersymmetry" context where (6.3) is satisfied.

One might wonder how the absence of massless matter charged under the $\mathrm{U}(1)$ factor is reconciled with charge completeness, which we have proven using Poincaré duality. To demonstrate how this is possible we carry out the Poincaré duality analysis of divisors and curves a little further in this case. As described in section 3.2, we begin by projecting out the divisors $\sigma_{\infty}, \pi^{*} C$ and the curves $F, \hat{C}$. In the case of the generic Schoen manifold $X$ there are 8 further sections $\sigma_{i}, i=1, \ldots, 8$, where the Mordell-Weil group $\mathbb{Z}^{8}$ is generated by $\sigma_{i}-\sigma_{\infty}$. Subtracting off $\sigma_{\infty}$ from these sections ensures that we have divisors that are orthogonal to the curve associated with the generic fiber $F$. We must further orthogonalize these divisors by subtracting appropriate multiples of the divisors $\pi^{*} C_{\alpha}$ so that they are orthogonal to the curves $\hat{C}_{\beta}$. This can be done by taking the divisors

$$
D_{i}=\sigma_{i}-\sigma_{\infty}-\pi^{*} K .
$$

From Poincaré duality, we expect that there are fibral curves on $X$, even after the above projections, that have nonzero inner product with $D_{i}$, associated with matter fields in the $6 \mathrm{D}$ theory that are charged under each of the $\mathrm{U}(1)$ factors. We can identify some such curves by starting with $\pi^{*} C_{\alpha} \cdot \sigma_{i}$ and orthogonalizing with respect to the divisors $\sigma_{\infty}, \pi^{*} C_{\beta}$. The resulting curves are of the form $\pi^{*} C \cdot\left(\sigma_{i}-\sigma_{\infty}\right)+\left(K_{B} \cdot C\right) F$. Note that these are fibral curves in homology but are expressed as linear combinations of holomorphic curves that are horizontal in the elliptic fibration, and do not have vanishing volume in the F-theory limit, so that these correspond to massive particles in the $6 \mathrm{D}$ F-theory model

\footnotetext{
${ }^{17}$ The generic Schoen manifold has an infinite number of distinct elliptic fibrations, see e.g. [102] for a recent analysis in the context of F-theory.
} 
that are charged under the $\mathrm{U}(1)$ factor associated with divisor $D_{i}$. In this way, we can satisfy charge completeness but not massless charge sufficiency for these non-Higgsable $\mathrm{U}(1)$ factors that satisfy (6.3). Note, however, that the inner product between such a curve and $D_{i}$ is even and given by $2 K_{B} \cdot C$. So we have not given a complete set of divisors and dual curves. Other curves may for example be associated with the lift of a curve in the base to a section that is only defined over that curve and not globally over the base; it is also possible that the curves we have identified here are even in integer homology, and thus there exist curves associated with half of these classes. We do not attempt a complete analysis of the set of allowed curves here.

\subsection{Nonabelian factors with a single adjoint matter field}

The non-Higgsable $\mathrm{U}(1)$ factors described in the previous subsection are closely related to nonabelian factors that satisfy (6.3). In particular, given any nonabelian factor with only a single adjoint field, we can Higgs the nonabelian factor to a product of U(1)s under which there is no charged matter. For example, given an $\mathrm{SU}(2)$ factor with a single adjoint we can turn on a diagonal expectation value for the adjoint reducing to a single $\mathrm{U}(1)$ under which no matter is charged. Geometrically, such nonabelian gauge factors arise in the Schoen construction when one of the factors in the fiber product of rational elliptic surfaces has degenerate fibers. The set of possible degenerate fibers for a rational elliptic surface is known and was tabulated in [103, 104]. We can also identify the gauge algebras that can appear on a divisor satisfying (6.3) from the geometry of rational elliptic surfaces. The intersection form on $d P_{9}$ is $U \oplus E_{8}$. An elliptic Calabi-Yau threefold with base $d P_{9}$ is generally a fiber product of rational elliptic surfaces. The elliptic fiber of the surface with which we take the fiber product removes the $U$ factor from the intersection form. This shows that the only gauge algebras we can realize are those with a lattice that is a sublattice of the $E_{8}$ lattice. Furthermore, this gives information about the kinds of discrete Mordell-Weil torsion that can be realized even in these exceptional cases.

As an example, consider what happens in the $\mathbb{Z}_{2}$ torsion case described by the Weierstrass model with (4.4) when the base is a generic rational elliptic surface $d P_{9}$. In this case, $\left[\alpha_{4}\right]=-4 K_{B}$, and $K_{B}$ is the class of the fiber with $K_{B} \cdot K_{B}=0$. This means that $\alpha_{4}$ is reducible and factorizes as $\alpha_{4}=\beta_{1} \gamma_{1} \delta_{1} \epsilon_{1}$. The gauge group then becomes $\mathrm{SU}(2)^{4} / \mathbb{Z}_{2}$. The presence of the $\mathbb{Z}_{2}$ quotient can be understood from the fact that if $x, y, z, t$ are orthogonal elements of the $E_{8}$ lattice each with self-intersection -2 , then $(x+y+z+t) / 2$ is also an element of the lattice with the same self-intersection since $E_{8}$ is unimodular.

The upshot of this is that for the exceptional cases satisfying (6.3), the presence of Mordell-Weil torsion is determined by different considerations to the general $6 \mathrm{D} \mathcal{N}=(1,0)$ gauge factor where we believe massless charge sufficiency holds. In some cases there is no Mordell-Weil torsion, though in others there is. Another example of a case with MordellWeil torsion with a rational elliptic surface base is the case of $\mathbb{Z}_{5}$ torsion (4.27). In this case we see that one can have two $\mathrm{SU}(5)$ factors each on a divisor of class $-K_{B}$, with global gauge group $(\mathrm{SU}(5) \times \mathrm{SU}(5)) / \mathbb{Z}_{5}$. 


\section{Conclusions}

In this paper we have used Poincaré duality on an elliptic Calabi-Yau threefold to prove that the charge completeness hypothesis for the connected component of the gauge group of every $6 \mathrm{D} \mathcal{N}=(1,0)$ supergravity theory that is realized in F-theory is equivalent to the standard understanding [27-29] of how the global form of the gauge group is encoded in F-theory geometry. The same result holds for every $4 \mathrm{D} \mathcal{N}=1 \mathrm{~F}$-theory model, for all charges under the connected component of the gauge group that arises from 7-branes and from sections of the F-theory elliptic fibration.

We have conjectured a stronger condition for $6 \mathrm{D}$ supergravity theories, that the massless charged states generate the full charge lattice of the theory whenever the anomaly coefficients satisfy $-a \cdot b>0$, which holds in all but a small set of exceptional cases. We have checked this for essentially all single-factor nonabelian gauge groups, and analyzed a large number of examples that illustrate the connection between the fundamental group of the gauge group of the supergravity theory and Mordell-Weil torsion in the F-theory picture. We have found that the only possible torsion subgroups that can arise without codimension two $(4,6)$ loci associated with superconformal sectors are $\mathbb{Z}_{2}, \mathbb{Z}_{3}, \mathbb{Z}_{4}$, and $\mathbb{Z}_{2} \oplus \mathbb{Z}_{2}$, except in the exceptional cases that violate massless charge sufficiency. We have given one example of an abelian U(1) theory that satisfies the massless charge sufficiency condition in a nontrivial way, but a more thorough analysis of more complicated U(1) theories with higher apparent charges is left as an open question.

An interesting feature we have identified in several of the models with fixed MordellWeil torsion groups is the appearance of SCFTs at points where anomaly cancellation and the discrete torsion structure suggest specific types of massless matter. In one case, corresponding to a situation previously analyzed in [91, 92], we find loci associated with $\left(E_{7} \times \mathrm{SU}(2)\right) / \mathbb{Z}_{2}$ matter in the $(\mathbf{5 6}, \mathbf{2})$ representation. These structures suggest that there may be a general way of understanding certain exotic matter structures in terms of local SCFTs in the F-theory context, as also suggested in [92]. This would be interesting to investigate further.

It would be nice to find some way to prove the charge completeness and massless charge sufficiency hypotheses for general 6D supergravity theories, extending the corresponding results of $[26]$ to flat space theories in $6 \mathrm{D}$.

Even in F-theory, we do not have a proof of charge completeness for theories with discrete gauge groups. Poincaré duality does not seem to be adequate to generalize the proof to such situations. We leave this interesting question, and a further analysis of 4D theories, for further work.

While we have analyzed a broad range of examples, the appearance of Mordell-Weil torsion in precisely those cases where the massless charge sufficiency conjecture predicts it should arise seems somewhat surprising and mysterious. It seems there should be some more general principle that guarantees that the global gauge group indeed matches the massless matter when $-a \cdot b>0$. It would be good to find a more fundamental understanding of this result, and to better understand the exceptional cases that violate the massless charge sufficiency condition when $-a \cdot b \leq 0$. Even lacking a proof of the massless 
charge sufficiency hypothesis for $6 \mathrm{D}$ theories, it would be interesting to find arguments based on basic principles of quantum gravity such as using black holes, along the lines of arguments in e.g. [25].

\section{Acknowledgments}

We would like to thank Paul Aspinwall, Daniel Harlow, Patrick Jefferson, Remke Kloosterman, Nikhil Raghuram, Andrew Turner, and Timo Weigand for helpful discussions. WT would like to thank the Kavli Institute for Theoretical Physics for hospitality on several visits during the progress of this project. We would also like to thank the Aspen Center for Physics for hospitality during the early and final stages of this project, and the Witwatersrand (Wits) rural facility and the MIT International Science and Technology Initiatives (MISTI) MIT-Africa-Imperial College seed fund program for hospitality and support during the latter stages of this project. The work of DRM was supported in part by National Science Foundation grant PHY-1620842 (U.S.A.). The work of WT was supported by the DOE under contract \#DE-SC00012567. This research was also supported in part by NSF grant No. PHY-1748958.

\section{A The fundamental group of the gauge group}

In this appendix, we will review some aspects of the topology of gauge groups, and describe how that topology is connected to the representation theory of the gauge group, in particular explaining why the fundamental group of the gauge group gives a reasonable measurement of the representation theory. Our primary references will be [105] and [106].

The gauge group $G$ of a physical theory is a compact Lie group which in general might not be connected. Let $G^{0}$ be the connected component of $G$ containing the origin. Then $G^{0}$ is a normal subgoup of $G$, and there is a short exact sequence of groups

$$
1 \rightarrow G^{0} \rightarrow G \rightarrow G / G^{0} \rightarrow 1
$$

where 1 denotes the trival group, and where $G / G^{0}=\pi_{0}(G)$ is a discrete group, the group of components of $G$. As in the body of this paper, we ignore questions about this discrete group, and only consider the connected subgroup $G^{0}$.

It is known [105, p. 70] that a compact Lie group is connected if and only if it is path-connected. So $G^{0}$ is also path-connected.

Theorem A.1 ([106, theorem V(8.1)]). A compact connected Lie group $G^{0}$ possesses a finite (unramified) cover which is isomorphic to the direct product of a compact simply connected Lie group $G_{0}$ and a (real) torus ${ }^{18} \boldsymbol{T} \cong \mathrm{U}(1)^{r}$.

Ref. [106] goes on to note that the covering map is given by the quotient by a finite central subgroup $\Xi$, namely:

$$
G^{0}=\left(G_{0} \times \boldsymbol{T}\right) / \Xi
$$

\footnotetext{
${ }^{18}$ In this appendix, we follow the conventions of the math literature and refer to this torus as $\boldsymbol{T}$; in the body of the paper, we have often remained closer to the physics literature and called the torus $\mathrm{U}(1)^{r}$.
} 
where $\Xi$ is a finite subgroup of the center $Z_{0} \times \boldsymbol{T}$ of $G_{0} \times \boldsymbol{T}$. Moreover, $G_{0}$ is a product of simply connected simple compact groups $G_{i}$

$$
G_{0}=\prod_{i=1}^{k} G_{i}
$$

and the center of $G_{0} \times \boldsymbol{T}$ is $\left(\prod_{i=1}^{k} Z_{i}\right) \times \boldsymbol{T}$, where $Z_{i}$ is the (finite) center of $G_{i}$. These centers are closely related to the representation theory.

Before continuing with the general theory, we pause to discuss two familiar examples. If $G^{0}=\mathrm{U}(n)$, the finite cover in question is obtained by extracting an $n^{\text {th }}$ root of the determinant. Then $(\operatorname{det} A)^{-1 / n} A \in \mathrm{SU}(n)$ for $A \in \mathrm{U}(n)$ and we can describe an element of $\mathrm{U}(1) \times \mathrm{SU}(n)$ as $\left((\operatorname{det} A)^{1 / n},(\operatorname{det} A)^{-1 / n} A\right)$. There is an order $n$ ambiguity here (the choice of $n^{\text {th }}$ root $)$ and so $\mathrm{U}(n) \cong(\mathrm{U}(1) \times \mathrm{SU}(n)) /(\mathbb{Z} / n \mathbb{Z})$, where the action of the central subgroup $\Xi \cong \mathbb{Z} / n \mathbb{Z}$ is generated by $\left(e^{-2 \pi i / n}, \operatorname{diag}\left(e^{2 \pi i / n}, \ldots, e^{2 \pi i / n}\right)\right)$. A representation of $\mathrm{U}(1) \times \mathrm{SU}(n)$ descends to a representation of $\mathrm{U}(n)$ if and only if the group $\Xi$ acts trivially within the given representation.

As a second example, let $G^{0}$ be the gauge group of the standard model. ${ }^{19}$ We take $G^{0}$ to be the subgroup of $\mathrm{SU}(5)$ consisting of matrices in block $2 \times 2$ and $3 \times 3$ form. (The fact that the matter representation of the standard model is compatible with this description is at the mathematical heart of the Georgi-Glashow grand unified model [108].) That is, a gauge group element consists of a unitary $2 \times 2$ matrix $A$ and a unitary $3 \times 3$ matrix $B$ such that the $5 \times 5$ matrix

$$
\left[\begin{array}{ll}
A & 0 \\
0 & B
\end{array}\right]
$$

has determinant 1, i.e., $(\operatorname{det} A)(\operatorname{det} B)=1$. To describe this group in terms of $\mathrm{SU}(2)$ and $\mathrm{SU}(3)$, we need a square root of $\operatorname{det} A$ and a cube root of $\operatorname{det} B$; since these determinants were inverses of each other, we need a sixth root of the common quantity.

That is, we can describe our group $G^{0}$ as a finite quotient of $\mathrm{U}(1) \times \mathrm{SU}(2) \times \mathrm{SU}(3)$, where the map to $\mathrm{SU}(5)$ is given by

$$
(\lambda, \alpha, \beta) \mapsto\left[\begin{array}{cc}
\lambda^{3} \alpha & 0 \\
0 & \lambda^{-2} \beta
\end{array}\right] .
$$

It is easy to see that the kernel of this map is $\Xi \cong \mathbb{Z} / 6 \mathbb{Z}$ generated by

$$
\left(e^{-2 \pi i / 6}, \operatorname{diag}\left(e^{6 \pi i / 6}, e^{6 \pi i / 6}\right), \operatorname{diag}\left(e^{-4 \pi i / 6}, e^{-4 \pi i / 6}, e^{-4 \pi i / 6}\right)\right) .
$$

Thus, $G^{0} \cong(\mathrm{U}(1) \times \mathrm{SU}(2) \times \mathrm{SU}(3)) /(\mathbb{Z} / 6 \mathbb{Z})$. The key fact here is that $\Xi$ acts trivially on all representations ocurring in the standard model, and it is the largest subgroup of $\mathrm{U}(1) \times \mathrm{SU}(2) \times \mathrm{SU}(3)$ to do so.

\footnotetext{
${ }^{19}$ The global structure of this group has various subtleties, and the corresponding representation theory is described in great detail in [107].
} 
Returning to the general case: the connected part $G^{0}$ of the gauge group will act on the various fields of the physical theory, ${ }^{20}$ and the charges under this group are determined by the charges under a maximal (compact) torus $H \subset G^{0}$. We can write this torus as

$$
H=\left(H_{0} \times \boldsymbol{T}\right) / \Xi \subset\left(G_{0} \times \boldsymbol{T}\right) / \Xi
$$

where $H_{0} \subset G_{0}$ is a maximal torus of $G_{0}$. The possible charges are described as group homomorphisms $\vartheta: H \rightarrow \mathrm{U}(1)$, or, writing ${ }^{21} H=\mathfrak{h} / \pi_{1}(H)$, as group homomorphims $\vartheta: \pi_{1}(H) \rightarrow \mathbb{Z}$ (extended from $\pi_{1}(H)$ to $\mathfrak{h}$ by extending scalars from $\mathbb{Z}$ to $\mathbb{R}$ ).

The set of possible charges

$$
\Lambda_{w}=\operatorname{Hom}\left(\pi_{1}(H), \mathbb{Z}\right)
$$

forms the weight lattice (or charge lattice) inside the real vector space $\mathfrak{h}^{*}=\operatorname{Hom}(\mathfrak{h}, \mathbb{R})$, and each finite-dimensional representation involves a finite number of such weights, which are the simultaneous $H$-eigenvalues under the representation. (In this context, a lattice inside a real vector space is a free $\mathbb{Z}$-module generated by a basis of the vector space.) The dual of the weight lattice

$$
\Lambda_{c w}=\operatorname{Hom}\left(\Lambda_{w}, \mathbb{Z}\right)
$$

forms the coweight lattice which coincides with $\pi_{1}(H)$.

The weights which occur as simultaneous $H$-eigenvalues in the adjoint representation of $G^{0}$ are known as the roots of $G^{0}$, and span a sublattice $\Lambda_{r}$ of $\Lambda_{w}$ known as the root lattice. Notice that since $\boldsymbol{T}$ lies in the center of $G^{0}$, the adjoint representation of $G^{0}$ maps $\boldsymbol{T}$ to the identity, which implies that $\Lambda_{r}$ is a sublattice of $\left(\Lambda_{w}\right)_{0}=\operatorname{Hom}\left(\pi_{1}\left(H_{0}\right), \mathbb{Z}\right)$, the weight lattice of $G_{0}$. In fact, $\Lambda_{r}$ has finite index in $\left(\Lambda_{w}\right)_{0}$, and $\left(\Lambda_{w}\right)_{0} / \Lambda_{r} \cong \prod_{i=1}^{k} Z_{i}$, where $Z_{i}$ is the center of the simple factor $G_{i}$ of $G_{0}$.

To specify a representation of $G^{0}$, we specify a representation of each $G_{i}$ as well as a representation of $\boldsymbol{T}$, and tensor them together; this will give a representation of $G^{0}$ provided that $\Xi$ acts trivially. The corresponding representation of $G_{i}$ determines a representation of its center $Z_{i}$, and these - together with the representation of $\boldsymbol{T}$ - determine the action of $\Xi$.

The fundamental group of $G_{0} \times \boldsymbol{T}$ is

$$
\pi_{1}\left(G_{0} \times \boldsymbol{T}\right)=\pi_{1}(\boldsymbol{T})=\mathbb{Z}^{r}
$$

where $r=\operatorname{dim} \boldsymbol{T}$, and its universal cover is $G_{0} \times \mathbb{R}^{r}$, so there is an exact sequence of abelian groups

$$
0 \rightarrow \pi_{1}\left(G_{0} \times \boldsymbol{T}\right)=\mathbb{Z}^{r} \rightarrow \pi_{1}\left(G^{0}\right) \rightarrow \Xi \rightarrow 0 .
$$

Thus, a maximal free subgroup of $\pi_{1}\left(G^{0}\right)$ determines the coweights of representations, and the finite quotient $\Xi$ determines the compatibility conditions. When $\Xi$ does not act

\footnotetext{
${ }^{20}$ For simplicity, we take all fields to be complex-valued, so that tori act through multiplication by some phase. In the real case, a phase rotation in the complex $z$-plane can be replaced by a circle action in the real $(x, y)$-plane.

${ }^{21}$ Here, $\mathfrak{h}$ is the Lie algebra of $H$, which is a finite dimensional real vector space with trivial Lie bracket.
} 
on $\boldsymbol{T}$, then it is isomorphic to the torsion subgroup of $\pi_{1}\left(G^{0}\right)$; on the other hand, in the explicit examples described above where $\Xi$ acts nontrivially on $\boldsymbol{T}=\mathrm{U}(1), \pi_{1}\left(G^{0}\right)=\mathbb{Z}$ and there is no torsion subgroup.

Open Access. This article is distributed under the terms of the Creative Commons Attribution License (CC-BY 4.0), which permits any use, distribution and reproduction in any medium, provided the original author(s) and source are credited.

\section{References}

[1] M.B. Green and J.H. Schwarz, Anomaly Cancellation in Supersymmetric D $=10$ Gauge Theory and Superstring Theory, Phys. Lett. B 149 (1984) 117 [InSPIRE].

[2] D.J. Gross, J.A. Harvey, E.J. Martinec and R. Rohm, Heterotic String Theory. 1. The Free Heterotic String, Nucl. Phys. B 256 (1985) 253 [INSPIRE].

[3] D.J. Gross, J.A. Harvey, E.J. Martinec and R. Rohm, Heterotic String Theory. 2. The Interacting Heterotic String, Nucl. Phys. B 267 (1986) 75 [INSPIRE].

[4] C. Vafa, The String landscape and the swampland, hep-th/0509212 [INSPIRE].

[5] A. Adams, O. DeWolfe and W. Taylor, String universality in ten dimensions, Phys. Rev. Lett. 105 (2010) 071601 [arXiv: 1006.1352] [INSPIRE].

[6] H.-C. Kim, G. Shiu and C. Vafa, Branes and the Swampland, Phys. Rev. D 100 (2019) 066006 [arXiv: 1905.08261] [INSPIRE].

[7] E. Palti, The Swampland: Introduction and Review, Fortsch. Phys. 67 (2019) 1900037 [arXiv: 1903. 06239] [INSPIRE].

[8] C. Vafa, Evidence for F-theory, Nucl. Phys. B 469 (1996) 403 [hep-th/9602022] [InSPIRE].

[9] D.R. Morrison and C. Vafa, Compactifications of F-theory on Calabi-Yau threefolds. 1, Nucl. Phys. B 473 (1996) 74 [hep-th/9602114] [InSPIRE].

[10] D.R. Morrison and C. Vafa, Compactifications of F-theory on Calabi-Yau threefolds. 2, Nucl. Phys. B 476 (1996) 437 [hep-th/9603161] [INSPIRE].

[11] L. Bhardwaj, D.R. Morrison, Y. Tachikawa and A. Tomasiello, The frozen phase of F-theory, JHEP 08 (2018) 138 [arXiv:1805.09070] [INSPIRE].

[12] V. Kumar and W. Taylor, String Universality in Six Dimensions, Adv. Theor. Math. Phys. 15 (2011) 325 [arXiv:0906.0987] [INSPIRE].

[13] V. Kumar, D.R. Morrison and W. Taylor, Global aspects of the space of $6 D N=1$ supergravities, JHEP 11 (2010) 118 [arXiv:1008.1062] [INSPIRE].

[14] W. Taylor and A.P. Turner, An infinite swampland of U(1) charge spectra in $6 D$ supergravity theories, JHEP 06 (2018) 010 [arXiv: 1803.04447] [INSPIRE].

[15] N. Seiberg and W. Taylor, Charge Lattices and Consistency of 6D Supergravity, JHEP 06 (2011) 001 [arXiv:1103.0019] [INSPIRE].

[16] S. Monnier and G.W. Moore, Remarks on the Green-Schwarz Terms of Six-Dimensional Supergravity Theories, Commun. Math. Phys. 372 (2019) 963 [arXiv:1808.01334] [INSPIRE]. 
[17] S.-J. Lee and T. Weigand, Swampland Bounds on the Abelian Gauge Sector, Phys. Rev. D 100 (2019) 026015 [arXiv: 1905.13213] [InSPIRE].

[18] C. Angelantonj, Q. Bonnefoy, C. Condeescu and E. Dudas, String Defects, Supersymmetry and the Swampland, JHEP 11 (2020) 125 [arXiv:2007.12722] [INSPIRE].

[19] N. Raghuram, W. Taylor and A.P. Turner, Automatic enhancement in 6D supergravity and F-theory models, JHEP 07 (2021) 048 [arXiv: 2012.01437] [INSPIRE].

[20] H.-C. Tarazi and C. Vafa, On The Finiteness of $6 d$ Supergravity Landscape, arXiv:2106.10839 [INSPIRE].

[21] A. Adams, N. Arkani-Hamed, S. Dubovsky, A. Nicolis and R. Rattazzi, Causality, analyticity and an IR obstruction to UV completion, JHEP 10 (2006) 014 [hep-th/0602178] [INSPIRE].

[22] H. Ooguri and C. Vafa, On the Geometry of the String Landscape and the Swampland, Nucl. Phys. B 766 (2007) 21 [hep-th/0605264] [INSPIRE].

[23] N. Arkani-Hamed, L. Motl, A. Nicolis and C. Vafa, The String landscape, black holes and gravity as the weakest force, JHEP 06 (2007) 060 [hep-th/0601001] [INSPIRE].

[24] J. Polchinski, Monopoles, duality, and string theory, Int. J. Mod. Phys. A 19S1 (2004) 145 [hep-th/0304042] [INSPIRE].

[25] T. Banks and N. Seiberg, Symmetries and Strings in Field Theory and Gravity, Phys. Rev. D 83 (2011) 084019 [arXiv:1011.5120] [InSPIRE].

[26] D. Harlow and H. Ooguri, Symmetries in quantum field theory and quantum gravity, Commun. Math. Phys. 383 (2021) 1669 [arXiv: 1810.05338] [INSPIRE].

[27] P.S. Aspinwall and D.R. Morrison, Nonsimply connected gauge groups and rational points on elliptic curves, JHEP 07 (1998) 012 [hep-th/9805206] [INSPIRE].

[28] P.S. Aspinwall, S.H. Katz and D.R. Morrison, Lie groups, Calabi-Yau threefolds, and F-theory, Adv. Theor. Math. Phys. 4 (2000) 95 [hep-th/0002012] [InSPIRE].

[29] C. Mayrhofer, D.R. Morrison, O. Till and T. Weigand, Mordell-Weil Torsion and the Global Structure of Gauge Groups in F-theory, JHEP 10 (2014) 016 [arXiv:1405.3656] [INSPIRE].

[30] F. Apruzzi, M. Dierigl and L. Lin, The Fate of Discrete 1-Form Symmetries in 6d, arXiv: 2008.09117 [INSPIRE].

[31] M. Cvetič, M. Dierigl, L. Lin and H.Y. Zhang, String Universality and Non-Simply-Connected Gauge Groups in 8d, Phys. Rev. Lett. 125 (2020) 211602 [arXiv: 2008.10605] [INSPIRE].

[32] M.B. Green, J.H. Schwarz and P.C. West, Anomaly Free Chiral Theories in Six-Dimensions, Nucl. Phys. B 254 (1985) 327 [InSPIRE].

[33] A. Sagnotti, A Note on the Green-Schwarz mechanism in open string theories, Phys. Lett. B 294 (1992) 196 [hep-th/9210127] [INSPIRE].

[34] J. Erler, Anomaly cancellation in six-dimensions, J. Math. Phys. 35 (1994) 1819 [hep-th/9304104] [INSPIRE].

[35] V. Kumar and W. Taylor, A Bound on 6D N=1 supergravities, JHEP 12 (2009) 050 [arXiv:0910.1586] [INSPIRE]. 
[36] V. Kumar, D.S. Park and W. Taylor, 6D supergravity without tensor multiplets, JHEP 04 (2011) 080 [arXiv: 1011.0726] [INSPIRE].

[37] A. Grassi and D.R. Morrison, Anomalies and the Euler characteristic of elliptic Calabi-Yau threefolds, Commun. Num. Theor. Phys. 6 (2012) 51 [arXiv:1109.0042] [InSPIRE].

[38] D.S. Park and W. Taylor, Constraints on $6 D$ Supergravity Theories with Abelian Gauge Symmetry, JHEP 01 (2012) 141 [arXiv:1110.5916] [INSPIRE].

[39] D.S. Park, Anomaly Equations and Intersection Theory, JHEP 01 (2012) 093 [arXiv:1111.2351] [INSPIRE].

[40] S. Monnier, G.W. Moore and D.S. Park, Quantization of anomaly coefficients in $6 D$ $\mathcal{N}=(1,0)$ supergravity, JHEP 02 (2018) 020 [arXiv:1711.04777] [INSPIRE].

[41] N. Seiberg and E. Witten, Comments on string dynamics in six-dimensions, Nucl. Phys. B 471 (1996) 121 [hep-th/9603003] [INSPIRE].

[42] J.J. Heckman, D.R. Morrison and C. Vafa, On the Classification of 6D SCFTs and Generalized ADE Orbifolds, JHEP 05 (2014) 028 [Erratum JHEP 06 (2015) 017] [arXiv:1312.5746] [INSPIRE].

[43] M. Del Zotto, J.J. Heckman, A. Tomasiello and C. Vafa, 6d Conformal Matter, JHEP 02 (2015) 054 [arXiv: 1407.6359] [INSPIRE].

[44] D.R. Morrison, TASI lectures on compactification and duality, in proceedings of the Theoretical Advanced Study Institute in Elementary Particle Physics (TASI 99): Strings, Branes, and Gravity, Boulder, CO, U.S.A., 31 May-25 June 1999, pp. 653-719 [hep-th/0411120] [INSPIRE].

[45] F. Denef, Les Houches Lectures on Constructing String Vacua, in Les Houches 87, Elsevier B.V. (2008), pp. 483-610 [arXiv:0803.1194] [INSPIRE].

[46] W. Taylor, TASI Lectures on Supergravity and String Vacua in Various Dimensions, arXiv:1104.2051 [INSPIRE].

[47] T. Weigand, F-theory, PoS TASI2017 (2018) 016 [arXiv:1806.01854] [INSPIRE].

[48] D.R. Morrison, What is F-theory?, to appear.

[49] K. Kodaira, On compact analytic surfaces. II, Ann. Math. 77 (1963) 563.

[50] K. Kodaira, On compact analytic surfaces. III, Ann. Math. 78 (1963) 1.

[51] V. Sadov, Generalized Green-Schwarz mechanism in F-theory, Phys. Lett. B 388 (1996) 45 [hep-th/9606008] [INSPIRE].

[52] A. Grassi and D.R. Morrison, Group representations and the Euler characteristic of elliptically fibered Calabi-Yau threefolds, J. Algebr. Geom. 12 (2003) 321 [math/0005196] [INSPIRE].

[53] V. Kumar, D.R. Morrison and W. Taylor, Mapping $6 D N=1$ supergravities to F-theory, JHEP 02 (2010) 099 [arXiv:0911.3393] [INSPIRE].

[54] T.W. Grimm and A. Kapfer, Anomaly Cancelation in Field Theory and F-theory on a Circle, JHEP 05 (2016) 102 [arXiv: 1502.05398] [INSPIRE].

[55] F. Bonetti and T.W. Grimm, Six-dimensional $(1,0)$ effective action of F-theory via M-theory on Calabi-Yau threefolds, JHEP 05 (2012) 019 [arXiv:1112.1082] [INSPIRE]. 
[56] L. Lin and T. Weigand, Towards the Standard Model in F-theory, Fortsch. Phys. 63 (2015) 55 [arXiv: 1406.6071] [INSPIRE].

[57] M. Cvetič, D. Klevers, D.K.M. Peña, P.-K. Oehlmann and J. Reuter, Three-Family Particle Physics Models from Global F-theory Compactifications, JHEP 08 (2015) 087 [arXiv: 1503.02068] [INSPIRE].

[58] C. Lawrie, S. Schäfer-Nameki and J.-M. Wong, F-theory and All Things Rational: Surveying U(1) Symmetries with Rational Sections, JHEP 09 (2015) 144 [arXiv:1504.05593] [INSPIRE].

[59] T.W. Grimm, A. Kapfer and D. Klevers, The Arithmetic of Elliptic Fibrations in Gauge Theories on a Circle, JHEP 06 (2016) 112 [arXiv:1510.04281] [INSPIRE].

[60] L. Lin and T. Weigand, G4-flux and standard model vacua in F-theory, Nucl. Phys. B 913 (2016) 209 [arXiv: 1604.04292] [inSPIRE].

[61] M. Cvetič and L. Lin, The Global Gauge Group Structure of F-theory Compactification with $\mathrm{U}(1) s$, JHEP 01 (2018) 157 [arXiv: 1706.08521] [INSPIRE].

[62] M. Cvetič, L. Lin, M. Liu and P.-K. Oehlmann, An F-theory Realization of the Chiral MSSM with $\mathbb{Z}_{2}$-Parity, JHEP 09 (2018) 089 [arXiv: 1807.01320] [INSPIRE].

[63] M. Cvetič, J. Halverson, L. Lin, M. Liu and J. Tian, Quadrillion F-Theory Compactifications with the Exact Chiral Spectrum of the Standard Model, Phys. Rev. Lett. 123 (2019) 101601 [arXiv: 1903.00009] [INSPIRE].

[64] W. Taylor and A.P. Turner, Generic Construction of the Standard Model Gauge Group and Matter Representations in F-theory, Fortsch. Phys. 68 (2020) 2000009 [arXiv:1906.11092] [INSPIRE].

[65] N. Raghuram, W. Taylor and A.P. Turner, General F-theory models with tuned $(\mathrm{SU}(3) \times \mathrm{SU}(2) \times \mathrm{U}(1)) / \mathbb{Z}_{6}$ symmetry, JHEP $04(2020) 008$ [arXiv:1912.10991] [INSPIRE]

[66] M. Bershadsky, K.A. Intriligator, S. Kachru, D.R. Morrison, V. Sadov and C. Vafa, Geometric singularities and enhanced gauge symmetries, Nucl. Phys. B 481 (1996) 215 [hep-th/9605200] [INSPIRE].

[67] S.H. Katz and C. Vafa, Matter from geometry, Nucl. Phys. B 497 (1997) 146 [hep-th/9606086] [INSPIRE].

[68] D.R. Morrison and W. Taylor, Matter and singularities, JHEP 01 (2012) 022 [arXiv:1106.3563] [INSPIRE].

[69] D. Klevers, D.R. Morrison, N. Raghuram and W. Taylor, Exotic matter on singular divisors in F-theory, JHEP 11 (2017) 124 [arXiv:1706.08194] [INSPIRE].

[70] T.W. Grimm and W. Taylor, Structure in $6 D$ and $4 D N=1$ supergravity theories from F-theory, JHEP 10 (2012) 105 [arXiv: 1204.3092] [INSPIRE].

[71] E. Witten, Phase transitions in M-theory and F-theory, Nucl. Phys. B 471 (1996) 195 [hep-th/9603150] [INSPIRE].

[72] J. Tate, On the conjectures of Birch and Swinnerton-Dyer and a geometric analog, in Séminaire Nicolas Bourbak 1964/65-1965/66, W.A. Benjamin Publisher, Exp. No. 306, pp. $415-440$.

[73] T. Shioda, On elliptic modular surfaces, J. Math. Soc. Jpn. 24 (1972) 20.

[74] R. Wazir, Arithmetic on elliptic threefolds, Compos. Math. 140 (2004) 567. 
[75] D.R. Morrison and W. Taylor, Classifying bases for 6D F-theory models, Central Eur. J. Phys. 10 (2012) 1072 [arXiv:1201.1943] [INSPIRE].

[76] O. Zariski, The theorem of Riemann-Roch for high multiples of an effective divisor on an algebraic surface, Ann. Math. 76 (1962) 560.

[77] T. Bauer, M. Caibăr and G. Kennedy, Zariski decomposition: a new (old) chapter of linear algebra, Am. Math. Mon. 119 (2012) 25 [arXiv:0911.4500].

[78] G. Martini and W. Taylor, 6D F-theory models and elliptically fibered Calabi-Yau threefolds over semi-toric base surfaces, JHEP 06 (2015) 061 [arXiv:1404.6300] [INSPIRE].

[79] Y.-N. Wang, Tuned and Non-Higgsable U(1)s in F-theory, JHEP 03 (2017) 140 [arXiv:1611.08665] [INSPIRE].

[80] D.R. Morrison, D.S. Park and W. Taylor, Non-Higgsable Abelian gauge symmetry and F-theory on fiber products of rational elliptic surfaces, Adv. Theor. Math. Phys. 22 (2018) 177 [arXiv:1610.06929] [INSPIRE].

[81] W. Taylor and A.P. Turner, Generic matter representations in $6 D$ supergravity theories, JHEP 05 (2019) 081 [arXiv: 1901.02012] [INSPIRE].

[82] J.H. Silverman and J. Tate, Rational Points on Elliptic Curves, Springer (1992).

[83] J.H. Silverman, The Arithmetic of Elliptic Curves, Springer (1986).

[84] D. Kubert, Universal bounds on the torsion of elliptic curves, Proc. Lond. Math. Soc. 33 (1976) 193.

[85] N. Hajouji and P.-K. Oehlmann, Modular Curves and Mordell-Weil Torsion in F-theory, JHEP 04 (2020) 103 [arXiv: 1910.04095] [INSPIRE].

[86] B. Mazur, Modular curves and Eisenstein ideal, Publ. Math. IHÉS 47 (1977) 33.

[87] B. Mazur, Rational isogenies of prime degree, Invent. Math. 44 (1978) 129.

[88] N. Bourbaki, Groupes et algèbres de Lie, Hermann, Paris France (1968), chapters IV, V, VI.

[89] S. Katz, D.R. Morrison, S. Schäfer-Nameki and J. Sully, Tate's algorithm and F-theory, JHEP 08 (2011) 094 [arXiv: 1106.3854] [INSPIRE].

[90] L.B. Anderson, J. Gray, N. Raghuram and W. Taylor, Matter in transition, JHEP 04 (2016) 080 [arXiv:1512.05791] [INSPIRE].

[91] Y. Kimura, Unbroken $E_{7} \times E_{7}$ nongeometric heterotic strings, stable degenerations and enhanced gauge groups in F-theory duals, arXiv: 1902.00944 [INSPIRE].

[92] M. Cvetič, J.J. Heckman and L. Lin, Towards Exotic Matter and Discrete Non-Abelian Symmetries in F-theory, JHEP 11 (2018) 001 [arXiv:1806.10594] [INSPIRE].

[93] Y.-C. Huang and W. Taylor, Comparing elliptic and toric hypersurface Calabi-Yau threefolds at large Hodge numbers, JHEP 02 (2019) 087 [arXiv: 1805.05907] [INSPIRE].

[94] D.R. Morrison and D.S. Park, F-Theory and the Mordell-Weil Group of Elliptically-Fibered Calabi-Yau Threefolds, JHEP 10 (2012) 128 [arXiv: 1208.2695] [INSPIRE].

[95] D.R. Morrison and W. Taylor, Sections, multisections, and U(1) fields in F-theory, arXiv: 1404.1527 [INSPIRE].

[96] D. Klevers, D.K.M. Peña, P.-K. Oehlmann, H. Piragua and J. Reuter, F-Theory on all Toric Hypersurface Fibrations and its Higgs Branches, JHEP 01 (2015) 142 [arXiv : 1408.4808] [INSPIRE]. 
[97] N. Raghuram, Abelian F-theory Models with Charge-3 and Charge-4 Matter, JHEP 05 (2018) 050 [arXiv: 1711.03210] [INSPIRE].

[98] F.M. Cianci, D.K.M. Peña and R. Valandro, High U(1) charges in type IIB models and their F-theory lift, JHEP 04 (2019) 012 [arXiv:1811.11777] [INSPIRE].

[99] A. Collinucci, M. Fazzi, D.R. Morrison and R. Valandro, High electric charges in M-theory from quiver varieties, JHEP 11 (2019) 111 [arXiv:1906.02202] [INSPIRE].

[100] N. Raghuram and W. Taylor, Large U(1) charges in F-theory, JHEP 10 (2018) 182 [arXiv: 1809.01666] [INSPIRE].

[101] D.R. Morrison and W. Taylor, Toric bases for 6D F-theory models, Fortsch. Phys. 60 (2012) 1187 [arXiv: 1204.0283] [INSPIRE].

[102] L.B. Anderson, X. Gao, J. Gray and S.-J. Lee, Fibrations in CICY Threefolds, JHEP 10 (2017) 077 [arXiv: 1708.07907] [inSPIRE].

[103] U. Persson, Configurations of Kodaira fibers on rational elliptic surfaces, Math. Z. 205 (1990) 1.

[104] R. Miranda, Persson's list of singular fibers for a rational elliptic surface, Math. Z. 205 (1990) 191.

[105] B.C. Hall, Lie groups, Lie algebras, and representations: An elementary introduction, second edition, in Graduate Texts in Mathematics 222, Springer-Verlag, New York NY U.S.A. (2015).

[106] T. Bröcker and T. tom Dieck, Representations of compact Lie groups, in Graduate Texts in Mathematics 98, Springer-Verlag, New York NY U.S.A. (1995).

[107] J.C. Baez and J. Huerta, The Algebra of Grand Unified Theories, Bull. Am. Math. Soc. 47 (2010) 483 [arXiv: 0904.1556] [INSPIRE].

[108] H. Georgi and S.L. Glashow, Unity of All Elementary Particle Forces, Phys. Rev. Lett. 32 (1974) 438 [INSPIRE]. 